\title{
The neuropsychological outcomes of non-fatal strangulation in domestic and sexual violence: A systematic review
}

\author{
Helen Bichard ${ }^{1,2}$, Christopher Byrne ${ }^{2,3}$, Christopher W. N. Saville ${ }^{1}$, and Rudi Coetzer ${ }^{2,3}$ \\ ${ }^{1}$ North Wales Clinical Psychology Programme, Bangor University \\ ${ }^{2}$ North Wales Brain Injury Service, Betsi Cadwaladr University Health Board \\ ${ }^{3}$ School of Psychology, Bangor University
}

\author{
...when I have pluck'd the rose, \\ I cannot give it vital growth again. \\ It must needs wither: I'll smell it on the tree. \\ [He kisses her] \\ Ah balmy breath, that dost almost persuade \\ Justice to break her sword... \\ [He strangles her]
}

Othello, Act V Scene II

Address for correspondence: Helen Bichard, North Wales Clinical Psychology Programme, School of Psychology, Brigantia Building, Bangor University, Bangor LL57 2DG; email helen.bichard@wales.nhs.uk guidelines. 


\begin{abstract}
This paper reviews the neurological, cognitive, psychological, and behavioural outcomes of non-fatal strangulation and, given shared physiological mechanisms, asks whether the hypoxic-ischaemic literature can serve as a proxy. 27 empirical, peer-reviewed studies were found which met the inclusion criteria. Neurological consequences included loss of consciousness, indicating at least mild acquired brain injury, stroke, seizures, motor and speech disorders, and paralysis. Psychological outcomes included PTSD, depression, suicidality, and dissociation. Cognitive and behavioural sequelae were described less frequently, but included amnesia and compliance. Overall, the evidence suggested strangulation in domestic violence and sexual assault can share all the serious consequences of hypoxic-ischaemic injury, but carries additional neuropsychological burden. However, no papers used formal neuropsychological assessment: the majority were medical case studies, or based on self-report. There is therefore a need for further neuropsychological research, focusing on cognitive and behavioural outcomes, using standardised tools, and control groups where possible. This is urgent, given societal normalisation of strangulation, and consent to 'rough sex' being used as a legal defence. We also discuss broader implications: the popularity of the 'choking game' with teenagers, and carotid injuries within mixed martial arts.
\end{abstract}

\title{
Keywords
}

Intimate partner violence; sexual assault; choking; hypoxic-ischaemic; neurobehavioural 


\section{Introduction}

Brain injury within domestic and sexual violence is belatedly gaining academic, medical, and legal attention. This is welcome, given the scale of the problem. More than one in three women are victims of intimate partner violence (IPV; WHO, 2019), 44\% report sexual assault, and 20\% rape (McQuown et al., 2016). In the majority of sexual assaults, the perpetrator is the victim's partner, so there is a significant overlap between the two areas, termed Intimate Partner Sexual Violence (Bagwell-Grey, Messing \& Baldwin-White, 2015). Corrigan and colleagues (2003) reported loss of consciousness in 30\% of IPV cases in emergency rooms, indicating at least a mild brain injury, and $67 \%$ presenting with residual problems that could be neurological. This is perhaps unsurprising, given evidence showing over $90 \%$ of IPV survivors have injuries to the head, neck, and face (Banks, 2007). The risk of brain injury within IPV thus appears significantly higher, both in terms of percentage, and absolute numbers, than the risk in contact sports and military action, despite not having benefitted from the same degree of clinical and academic focus (Chapman \& Diaz-Arrastia, 2014; Koh, Cassidy \& Watkinson, 2003).

Within this new field, the research emphasis has been on traumatic brain injury (TBI). However, strangulation has emerged as a "hidden epidemic" (Taliaferro, Mills \& Walker, 2001, p.294). A systematic review reported the lifetime prevalence of strangulation to be between $3.0 \%$ and $9.7 \%$ in community-living adults; amongst women who are victims of systematic abuse, this rises to 50-68\% (Kwako et al., 2011; Sorenson, Joshi, \& Sivitz, 2014; Wilbur et al., 2001). Evidence indicates up to $17 \%$ of those strangled lose consciousness, indicating at least a mild TBI (Wilbur et al., 2001). Incidence is also high: in a US study of IPV/sexual assault health encounters, strangulation was reported in $23 \%$ of the assaults (McQuown et al., 2016). A similar UK audit noted strangulation in one in five cases 
presenting at a sexual assault referral centre (White, 2018). For incidence to be so high versus lifetime prevalence is suggestive of repeated injuries and, indeed, the literature confirms this, with studies showing half of survivors reporting being strangled between three and 20 times (Vella, 2013; Wilbur et al., 2001).

Although it is possible for a woman to strangle a man, as it can take more pressure to open a canned drink than to occlude the jugular vein (Green, 2017), strangulation appears to be a gendered crime. Sorenson and colleagues (2014) reports lifetime discrepancy between four and eleven-fold. In a review of 300 cases within the San Diego City Attorney’s Office, 298 involved a male perpetrator and a female victim (McClane, Strack \& Hawley, 2001). In White's SARC audit (2018), only two out of 70 victims were male. Indeed, a meta-analysis reviewing gender differences in violence stated strangulation "is very clearly a male act" (Archer, 2000, p. 327). There may be anatomical reasons behind this, in terms of hand and neck span, but the literature also suggests a power dynamic, often triggered by Othello-like jealousy, and a desire to assert control (Joshi, Thomas \& Sorenson, 2012; Sorenson et al., 2014). Thomas and colleagues (2014) describe strangulation's role as "setting the stage" (p.125): ensuring that it is understood that the main actor can or will kill.

Although strangulation can result in blunt force trauma to the neck, the method and physiological impact on the brain is different from most TBI. Strangulation can be defined as the external compression of the airway and/or blood vessels, leading to restricted oxygenated blood flow to, and deoxygenated blood from, the brain. This can be achieved with a ligature (garrotting), by body weight (throttling, or positional strangulation), or manually. Evidence largely gleaned from autopsies, and from assessing the risk of the 'choke hold' carotid restraint used by police, has been able to show the pathophysiology of strangulation, as set out below (Clarot, Vaz, Papin \& Proust, 2005; de Boos, 2019; Hawley, McClane \& Strack, 
2001; Monahan, Purushotham \& Biegon, 2019). Figure 1 serves as reference for the location of the relevant anatomical structures.

- Obstruction of the larynx cutting off airflow to the lungs (i.e. asphyxiation, leading to hypoxia), which may continue after pressure has been lifted if the neck structure has been damaged (e.g. hyoid fracture);

- Occlusion of the jugular veins, leading to venous congestion, increased intracranial pressure, decreased respiration, and possible pinpoint haemorrhage (petechiae);

- Occlusion of the internal carotid artery, restricting blood flow to the brain (i.e. ischaemic). This is more likely to happen when the attacker is facing the victim. If pressure is at base of neck, vertebral arteries may also be occluded. Again, this may continue once pressure has been removed if there has been arterial dissection;

- Triggering of the carotid sinus reflex, leading to dysrhythmia, possible cardiac arrest, and thus further lack of blood to the brain (hypoxic-ischaemic);

- Damage to the thyroid gland, resulting in possible 'thyroid storm', in which acute hyperthyroidism can cause congestive heart and multi-organ failure.

Figure 1. Schematic illustration of neck anatomy, indicating main structures vulnerable in strangulation

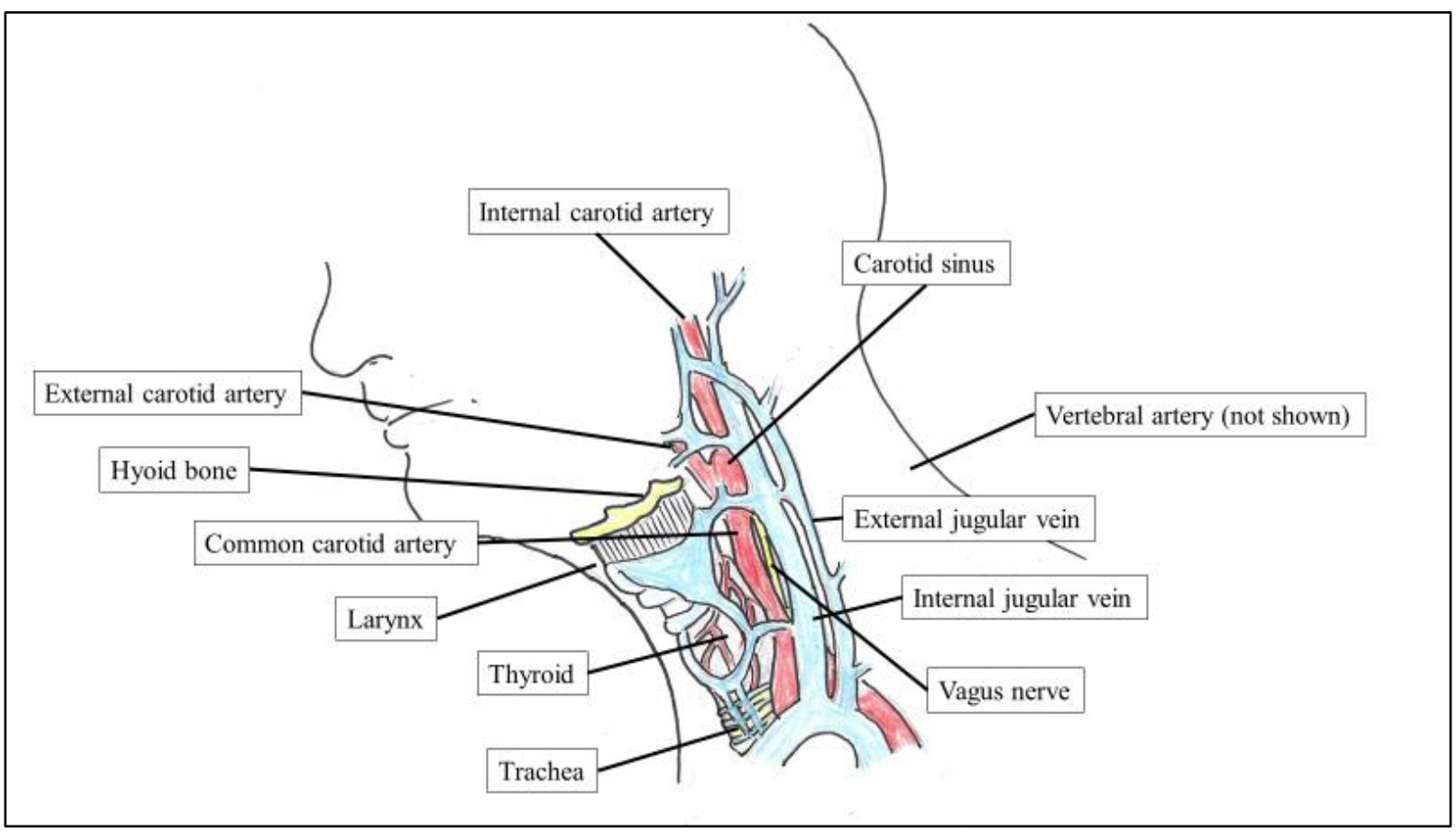


Any or all of these mechanisms could damage the brain, and quickly. In the notorious Red Wing studies (Kabat \& Anderson, 1943), in which psychiatric inmates and prisoners were strangled in order to increase the US military's understanding of why wartime airmen were blacking out, consciousness (and therefore memory of, and control over, events) was lost within four to ten seconds of arterial pressure, followed by anoxic seizures at six to eight seconds. Bladder control can be lost after 15 seconds, and bowels after 30, with decerebrate posturing after 20 seconds, indicating damage at a brain stem level, and, finally, brain death at between one and six minutes (de Boos, 2019). For asphyxiation only (so breathing prevented, but blood still flowing to the brain), the course depends on how much oxygen is present in the blood and lungs. Memories will still be formed while the person retains consciousness. Panic-induced struggling against the attacker may cause its own injuries. Eventually consciousness will be lost as the brain runs out of oxygen.

The relatively small amount of pressure required to affect the various injuries is sobering: 4.4lbs for the jugular, 11lbs for the carotid arteries, 33lbs for the trachea, and 66lbs for the vertebral arteries (Shields, Corey, Weakley-Jones \& Stewart, 2009). Figure 1 also highlights the proximity of the key structures; one can infer how difficult it would be to affect one in isolation. For those who survive strangulation, different brain areas react to a different time scale. Some parts of the brain stem and the hippocampus are known to be particularly vulnerable to lack of blood flow, along with the dentate nucleus, and the cerebellum (Hawley et al., 2001). Some cells may survive for days before dying, and the wider literature contains reports of stroke delayed by almost two weeks following other methods of carotid bisection (Levack, Pettitt, \& Winston, 2009). In fact, in a large study $(\mathrm{n}=300)$ only $39 \%$ had symptoms on the day of the injury (Strack, McClane \& Hawley, 2001). That Shakespeare depicts Desdemona regaining consciousness, and pleading with her assailant, before finally 
succumbing, is therefore not as ridiculous as literary critics have historically suggested (Cooper, 1949).

In addition to the neurological damage, leading to possible cognitive and behavioural changes, there is also the risk of significant psychological trauma. Strangulation has been called "the edge of homicide" (Strack \& Gwinn, 2011, p.32). If a woman has been strangled by her partner, the risk of attempted murder increases sevenfold, and death by a factor of eight (Glass et al., 2008). Not being able to breathe - air hunger - is a primal fear, experienced even in controlled laboratory tests (Banzett, Lansing, Evans \& Shea, 1996). In the uncontrolled IPV/sexual assault situation, the perpetrator, literally, has the woman's life in his hands; a woman who could well be his wife or girlfriend. He dictates whether she takes her next breath or not, and may, like Othello, have his gaze locked on her as she struggles. Strangulation is a uniquely intimate act of terrorism (Johnson, 2010), and it is not difficult to see why it could leave more than physical marks, such as PTSD and other trauma reactions. Attempts have already been made to synthesise our understanding of this new and important area. Pritchard and colleagues (2017) produced a helpful narrative review, outlining the history of the subject, with particular focus on the US legal response, where strangulation has now been reclassified as a felony. However, the search was not systematic, and the paper does not reference outcomes. An excellent integrative review (Patch, Anderson and Campbell, 2018) did follow PRISMA guidelines, but excluded studies before 2000, and was undertaken from a nursing perspective, requiring there to have been an emergency healthcare interaction. Given we know there is significant under-reporting, and the San Diego study suggested as few as 5\% of women will seek medical attention (Strack et al., 2001), it was felt that a more liberal and transdisciplinary approach might yield fuller findings. 
This review therefore aims to map the knowledge currently held within the medical, legal, social work, policing, psychological, and grey literature. Specifically, what evidence do we have for the neurological, cognitive, behavioural, and psychological impact of strangulation within IPV and sexual assault? Secondly, given the similar mechanics, is this any different from the documented outcomes of hypoxic-ischaemic brain injury (e.g. cardiac arrest), or can that body of literature be used as a proxy?

\section{Method}

The review was carried out according to PRISMA guidelines (Moher, Liberati, Tetzlaff \& Altman, 2009). It was registered in advance with PROSPERO, reference CRD42019160487.

\section{Search sources and strategy}

A deliberately wide selection of databases was used, given the transdisciplinary nature of the topic: PubMed, PsycINFO, CINHAL, Proquest, ASSIA, Web of Science, WestLaw, Open Grey, and Ethos. The rationale for including doctoral theses was that then a specific search could be conducted to ascertain whether a paper had been published based on the findings. The initial search consisted of paired combinations, based on titles and key words from other studies, reflecting the injury (strangulation) and the outcomes (brain injury and its sequelae). This was then joined with terms reflecting the context (IPV and sexual assault). The ProQuest script was:

\footnotetext{
((strangl* OR strangulation OR chok* OR "breath play" OR throttl* OR ligature OR garrot*) AND (neuro* OR asphyxi* OR cardiac OR Aneurysm OR stroke OR vascular OR "brain injury" OR "brain damage" OR hypox* OR anox* OR cogniti* OR psych* OR "mental health" OR emotion* OR dementia OR encephalopathy OR behavio* OR ischaemi*)) AND ("sexual assault" OR "sexual abuse" OR "spouse abuse" OR "spousal abuse" OR "partner abuse" OR "domestic violence" OR "sexual violence" OR "intimate partner violence" OR "intimate terrorism" OR "situational couple violence" OR batter* OR rape OR "rough sex" OR "dating violence")
} 
The search was conducted on the $17^{\text {th }}$ December, 2019.

\section{Eligibility criteria}

To qualify for inclusion, articles needed to be peer-reviewed, empirical papers, and refer to neurological, psychological, behavioural, or cognitive outcomes of strangulation (manual, ligature, or throttling) within domestic or sexual violence. No date limits were set, given the need for breadth. Exclusion criteria were: general violence not limited to IPV or sexual assault; focus on perpetrator; strangulation not separated out from other forms of violence; fatal; non-neurological outcomes only (e.g. neck lacerations, bruising); limited to policing or legal process; not adult or human; self-inflicted (e.g. auto-erotic asphyxia or hanging); no English version available.

\section{Quality assessment}

Based on specialist librarian advice, all studies were assessed using the suite of critical appraisal tools from the Joanna Briggs Institute (2017). Analytical cross sectional studies were measured against an eight item checklist, e.g. were confounding factors identified and controlled for, were outcomes measured in a valid and reliable way, and was appropriate statistical analysis undertaken. Case reports were appraised against a different eight item checklist, e.g. was there clear description of the patient's demographic characteristics and history, and were diagnostic tests and assessment methods specified. There was a 10-item checklist for qualitative research, e.g. congruity between research objectives and methodology, addressing the influence of the researcher on the research, and whether the conclusions drawn flowed from the analysis of the data. These are all checklists for inclusion in reviews, and are not intended to provide a formal grading system, or cut-off scores. However, our appraisal against the checklists has been included in the data extraction tables, 
with a higher score indicating that the authors have more closely adhered to methodological best practice.

\section{Risk of bias}

The first author conducted the search, and results were reviewed against these criteria and refined by the second author. Data on outcomes were extracted, and recorded in Table 1 by the first author. Comparison was made with the sequelae of hypoxic-ischaemic injuries as outlined by the International Brain Injury Association (Arciniegas, 2012). Again, outcome data and quality assessments were inspected and refined by the second author, and then the whole review was checked by the remaining authors, who were available in case of disagreement, but this was not necessary. 


\section{Results}

\section{Information extraction}

The initial search yielded 1,433 articles, with a further 43 added from reference lists.

Subsequent selection involved four main phases; see Figure 2. A total of 27 articles were included in the final sample.

Figure 2. PRISMA Flow Diagram
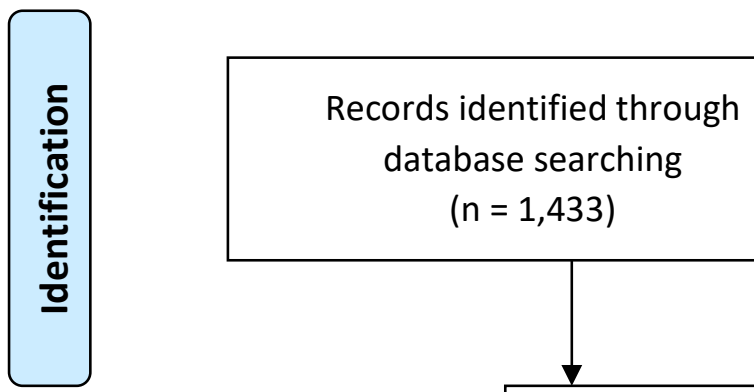

Records after duplicates removed

$(n=781)$
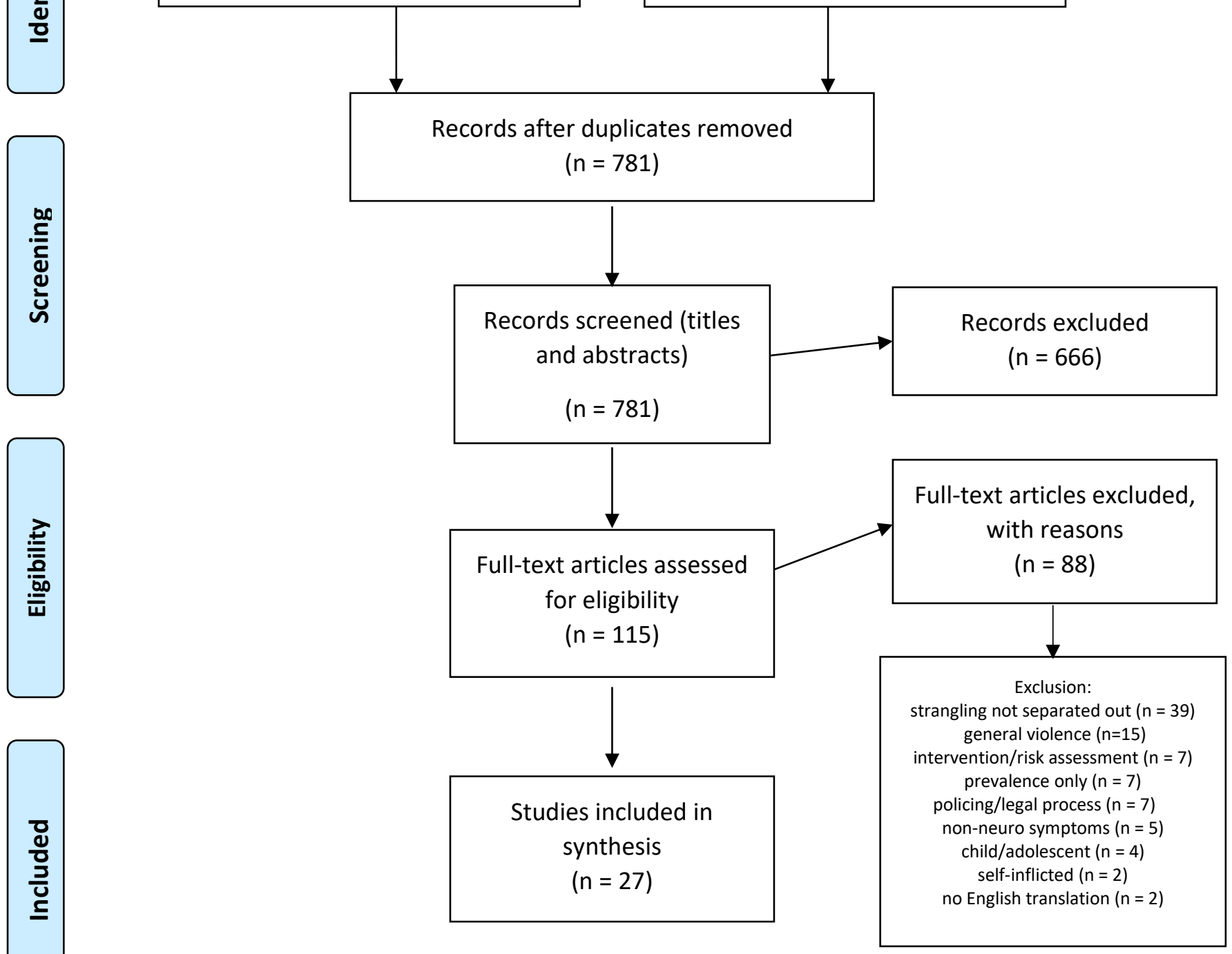

Additional records identified from reference lists

$$
(n=43)
$$

ild/adolescent $(n=4)$

self-inflicted $(n=2)$

no English translation $(n=2)$ 


\section{Analysis of the articles}

Table 1 provides key features of the final list of articles. They were published between 1980 and 2019 across 10 countries, with the USA being the main contributor, with 16/27.

\section{Characteristics of samples}

There were no randomised, population, or prospective samples. People were identified through their contact with the healthcare, police, or justice systems, or had been helped by IPV organisations. Sample size was bimodal, with nine case reports ( $\mathrm{N}=1$ to 3$)$, and eleven large retrospective analyses of existing records $(\mathrm{N}=102$ to 1,064$) .21 / 27$ studies had entirely female samples, and one study interviewed heterosexual couples with a male attacker. Of those studies where men had also been victims, they were in the minority, ranging from 2/14 (Yen et al., 2007) to 2/300 (Strack et al., 2001). Most studies reported a mean age in the early thirties. There were no controls. Joshi et al. (2012) and Thomas et al. (2014) tried to recruit IPV survivors who had not been strangled, but were unable. In the majority of studies (19/29) strangulation was in the context of IPV. 4/27 involved sexual assault, with partners being the assailant in two of those, and 4/27 recruited both IPV and sexual assault victims. Ralston and colleagues (2019) specifically excluded sexual assault.

\section{Study design and methodological considerations}

All studies were cross-sectional, although three case reports cited GP follow-up after several years. Alongside the nine clinical case reports written by the treating clinicians, there were another five analyses of hospital records, written by ED medics, forensic nurses, or radiologists, and three analyses of police and legal reports. There were no studies written from a neuropsychological perspective. These retrospective analyses depend on the depth and breadth of what was documented at the time, and who was doing this: for example, Strack et al. (2001) emphasise the inconsistency and paucity of symptom reporting by police, limited 
largely to visible injury. Similarly, using imaging data will be limited to what can be identified on a scan. Five studies were survey-based, and could therefore generate additional, transdisciplinary data. Of those studies which used statistical analysis, this was largely descriptive. Only four studies used inferential analysis, including t tests and odds ratios, chisquare, binomial/multinomial logistic regression, and risk ratios (Davins-Pujols et al., 2014; Messing et al., 2014 and 2018; Mittal et al., 2019). Where method was stated in the five qualitative studies, this was based on grounded theory. One used mixed methods, combining analysis of police records and follow-up depth interviews (Farr, 2002). The others used focus groups, and found this successful, despite the sensitivity of the topic.

\section{Assessment}

None of the studies used formal neuropsychological assessment. The majority of cognitive and psychological outcomes were based on participants' spontaneous self-reports. Shields et al. (2010) suggest this may underestimate complaints, given participants' tendency towards minimisation and denial, and possible amnesia resulting from strangulation. Where objective assessment was undertaken, this was guided by profession, and varied significantly. For example, Plattner and colleagues (2004) analysed hospital records of imaging results and clinical presentation, but note no evidence of neurological examination. There was no standard strangulation assessment tool identified, in either the medical or policing fields.

Two survey-based studies developed their own questions to record symptoms (Smith et al., 2001; Wilbur et al., 2001). Messing and colleagues $(2014,2018)$ and Mittal and colleagues (2019) were the only authors to used validated psychometrics, although none of these were strangulation-specific: Revised Conflict Tactics Scale (CTS2; Strauss et al., 1996), Danger Assessment Scale (Campbell et al., 2009), Women's Experience of Battering Scale (Smith, Earp, \& DeVellis, 1995), Primary Care Post-Traumatic Stress Disorder Screen (Cameron \& 
Gusman, 2003), Abuse Behaviour Inventory (Zink et al., 2007), Rosenberg Self-Esteen Scale (1965), and the Centre for Epidemiologic Studies Depression Scale (Radloff, 1977).

The timing of assessment differed. Case reports and retrospective record analyses tended to be based on immediate outcomes. There is therefore the risk that symptoms may not have developed, and victims were not yet in a position to report more specific difficulties. Where studies were delayed, by contrast, this relied on participants' recall, which may have been compromised by time, and amnesia for events, and many of the physical symptoms may have receded.

\section{Quality of evidence}

Qualitative researchers, despite intending to, found it difficult to recruit comparison groups who had not been strangled, given the high prevalence and incidence figures (Joshi et al., 2012; Thomas et al., 2014). However, Davins-Pujols et al. (2014), Messing et al. (2014, 2018), and Zilkens et al. (2016) were able to identify IPV victims not reporting strangulation to act as a comparison, and were the only studies thereby to attempt to control for confounding factors. Smith and colleagues (2001) were able to compare multiple to single strangulation events. There were only two clinical follow-ups by authors (Clarot et al., 2004; Malek et al, 2000), and one anecdotal report from the patient's GP after several years (Milligan \& Anderson, 1980). Only 3/27 studies used standardised psychometrics (Messing et al., 2014 \& 2018; Mittal et al., 2019). Overall therefore, the quality of the evidence would have been low if measured by standard grading tools. Using the JBI critical appraisal tools the median score for both cross sectional studies and case reports was 6/8. Quality was higher, albeit against different criteria, for the qualitative studies, although there was a widespread failure to frame the research in terms of relevant theory, or address the influence of the researcher on the research. 
Table 1. Key features of studies documenting the outcomes of non-fatal strangulation in Intimate Partner Violence and sexual assault

\begin{tabular}{|c|c|c|c|c|c|c|c|}
\hline Reference & Country & Study Design & Sample & Quality & Neuropathology & $\begin{array}{l}\text { Neuropsychological outcomes } \\
\text { (neurological, cognitive, emotional, } \\
\text { behavioural) }\end{array}$ & $\begin{array}{l}\text { Additional to hypoxic- } \\
\text { ischaemic? }\end{array}$ \\
\hline $\begin{array}{l}\text { Clarot, Vaz, Papin, \& } \\
\text { Proust (2004) }\end{array}$ & France & $\begin{array}{l}\text { Case report } \\
\text { (hospital) }\end{array}$ & $\begin{array}{l}\mathrm{N}=2 \text {, only } 1 \mathrm{IPV}- \\
\text { related } \\
42 \text { year old woman } \\
\text { strangled by husband }\end{array}$ & $\begin{array}{l}\text { JBI Case Reports: } \\
6 / 8 \\
\text { Nil for } 4 / 6\end{array}$ & $\begin{array}{l}\text { Bilateral common carotid } \\
\text { artery dissection with } 40 \% \\
\text { stenosis } \\
\text { CT scan normal }\end{array}$ & $\begin{array}{l}\text { Discharged from Emergency Department } \\
\text { following strangulation; returned two days } \\
\text { later, with severe headaches. } \\
\text { No neurologic sequelae at } 5 \text { month follow-up }\end{array}$ & $\begin{array}{l}\text { Pathological: Arterial } \\
\text { dissection }\end{array}$ \\
\hline $\begin{array}{l}\text { Davins-Pujols, } \\
\text { Salamero, Aznar- } \\
\text { Martinez, Alegret, \& } \\
\text { Perez-Testor (2014) }\end{array}$ & Spain & $\begin{array}{l}\text { Retrospective, } \\
\text { cross sectional } \\
\text { analysis of client } \\
\text { assessment data }\end{array}$ & $\begin{array}{l}\mathrm{N}=161 \\
\text { in specialised } \\
\text { psychology outpatient } \\
\text { service for abused } \\
\text { women; } \\
41 \text { women had been } \\
\text { strangled; mean age } \\
38.9\end{array}$ & $\begin{array}{l}\text { JBI Cross Sectional: } \\
7 / 8 \\
\text { Nil for } 6\end{array}$ & & $\begin{array}{l}\text { Women who were strangled significantly } \\
\text { more likely to experience feeling of danger } \\
\text { (OR } 9.74, p<0.001) \text { than other forms of } \\
\text { physical violence. }\end{array}$ & $\begin{array}{l}\text { Psychological: feeling } \\
\text { of danger, threat to life } \\
\text { from other }\end{array}$ \\
\hline De Boos (2019) & Australia & $\begin{array}{l}\text { Case report } \\
\text { (hospital) }\end{array}$ & $\begin{array}{l}\mathrm{N}=1 \\
21 \text { year old woman } \\
\text { strangled } 3 \text { times over } \\
20 \text { minutes by partner }\end{array}$ & $\begin{array}{l}\text { JBI Case Reports: } \\
7 / 8 \\
\text { Nil for } 4\end{array}$ & Tracheal perforation & $\begin{array}{l}\text { Pain } \\
\text { Dysphonia } \\
\text { Dysphagia } \\
\text { Confusion } \\
\text { Amnesia - could not articulate events } \\
\text { Nausea } \\
\text { Potential loss of consciousness } \\
\text { Feeling of being 'choked to death' } \\
\text { Lost to follow-up }\end{array}$ & $\begin{array}{l}\text { Pathological: } \\
\text { Perforated trachea } \\
\text { Neurological: } \\
\text { Pain } \\
\text { Dysphonia } \\
\text { Nausea } \\
\text { Psychological: } \\
\text { Existential threat }\end{array}$ \\
\hline $\begin{array}{l}\text { Eiskovits \& Winstok } \\
\text { (2002) }\end{array}$ & Israel & Qualitative & $\begin{array}{l}\mathrm{N}=48 \\
24 \text { in-depth } \\
\text { interviews with } \\
\text { heterosexual couples } \\
\text { who had stayed } \\
\text { together despite IPV; } \\
\text { one woman describes } \\
\text { strangulation. }\end{array}$ & $\begin{array}{l}\text { JBI Qualitative Research } \\
7 / 10 \\
\text { Nil for } 2 / 6 / 7\end{array}$ & & $\begin{array}{l}\text { Realisation of imminent death, and battle for } \\
\text { survival vs attacker: "Then I knew it's either } \\
\text { him or me" (p. 695) }\end{array}$ & $\begin{array}{l}\text { Psychological: } \\
\text { Existential fear and } \\
\text { survival response vs } \\
\text { attacker }\end{array}$ \\
\hline Farr (2002) & USA & $\begin{array}{l}\text { Mixed - content } \\
\text { analysis of police } \\
\text { reports, } \\
\text { qualitative } \\
\text { interviews }\end{array}$ & $\begin{array}{l}\mathrm{N}=30 \\
\text { survivors (all female) } \\
\text { of attempted } \\
\text { homicide; mean age } \\
34 ; 11 \text { were } \\
\text { choked/strangled; } 3 \\
\text { describe strangulation }\end{array}$ & $\begin{array}{l}\text { JBI Qualitative Research } \\
8 / 10 \\
\text { Nil for } 6 / 7\end{array}$ & & $\begin{array}{l}\text { "Being killed and watching it" (p. 275) - } \\
\text { dissociation? } \\
\text { Belief that they are about to die, one survivor } \\
\text { referring to having been "killed" when } \\
\text { "choked" (p. 275) } \\
\text { "Traumatic immobility" (p.275) }\end{array}$ & $\begin{array}{l}\text { Psychological: } \\
\text { Existential fear } \\
\text { Belief that did actually } \\
\text { 'die' } \\
\text { Dissociation } \\
\text { Traumatic immobility }\end{array}$ \\
\hline
\end{tabular}


Two survivors taken into public after

strangulation and no attempt made to esca

either due to fear, or because it never

Cognitive:

\begin{tabular}{|c|c|c|c|}
\hline $\begin{array}{l}\text { Funk \& Schuppel } \\
\text { (2003) }\end{array}$ & USA & $\begin{array}{l}\text { Case report } \\
\text { (hospital) }\end{array}$ & $\begin{array}{l}\mathrm{N}=1 \\
24 \text { year old woman } \\
\text { strangled three times } \\
\text { by partner whilst } 7 \\
\text { months pregnant }\end{array}$ \\
\hline
\end{tabular}

JBI Case Reports:

Nil for 6 initiation? occurred to them that they could (p. 276)
Right sub-conjunctival "I thought I would die"

haemorrhage

Petechiae right frontal region

"I thought I

Loss of consciousness

Headache

Ptosis

Pain

Dysphagia

Difficulty breathing

No follow-up
Agnosia/lack of

Pathological:

Petechiae, conjunctival

haemorrhage

Neurological:

Ptosis

Pain

Psychological: Existential fea
Joshi, Rahill, Lecano, \& Haiti Jean (2014)

Qualitative

\section{$\mathrm{N}=27$}

8 survivors of sexual

assault (age 19-45

years)

\section{BI Qualitative Research}

$9 / 10$

Nil for 7

\begin{tabular}{|c|c|c|c|}
\hline $\begin{array}{l}\text { Joshi, Thomas, \& } \\
\text { Sorenson (2012) }\end{array}$ & USA & Qualitative & $\begin{array}{l}\mathrm{N}=17 \\
\text { Women attending } \\
\text { domestic violence } \\
\text { shelter. Age } 21-47 \text {, } \\
14 \text { self-identified as } \\
\text { African American. } \\
\text { All strangled, } 15 / 17 \\
\text { multiple times. }\end{array}$ \\
\hline
\end{tabular}

\section{JBI Qualitative Research Petechiae}

$9 / 10$

Nil for 7
Stroke

S

"Dappiyanmp" used to describe sexual
assault (translates as ‘strangled like a
chicken'; p. 1635)
Headache
Tinnitus
Vision changes
Insomnia
Hypervigilance
Depression
Feelings of worthlessness and hopelessness
Suicidality
"I'm traumatised...I black out; you can be
talking to me right now and I can't see you,
can't hear you" (p. 1636) - dissociative
seizures?

Neurological:

Tinnitus

Insomnia

Psychological:

Hypervigilance

Depression

weelings of

hopelessness

hopelessness
Suicidality

Suicidality

Dissociative seizures?

$\begin{array}{ll}\text { 14/17 lost consciousness; } 2 \text { close to blacking } & \text { Pathological: } \\ \text { out } & \text { Petechiae } \\ \begin{array}{l}\text { Near death experience "life flashing before } \\ \text { eyes...saw my own face" (p. 9) }\end{array} & \text { Stroke } \\ \begin{array}{l}\text { Dysphasia } \\ \text { Dysphagia } \\ \text { Pain }\end{array} & \text { Neurological: } \\ \text { Incontinence } & \text { Incontinence } \\ \text { Tinnitus } & \text { Tinnitus } \\ \text { Physical weakness } & \text { Insomnia } \\ \text { Nightmares } & \text { Pain } \\ \text { Insomnia } & \\ \text { Anxiety } & \text { Psychological: } \\ \text { Suicidality } & \text { Nightmares } \\ & \text { Anxiety } \\ & \text { Fear }\end{array}$




\begin{tabular}{|c|c|c|c|c|c|c|c|}
\hline & & & & & & $\begin{array}{l}\text { Heightened and persistent fear } \\
\text { Exacerbation of existing mental health } \\
\text { problems } \\
\text { Triggering in new relationships } \\
\text { Minimisation of severity } \\
\text { Lack of medical help-seeking }\end{array}$ & $\begin{array}{l}\text { Exacerbated existing } \\
\text { mental health } \\
\text { difficulties } \\
\text { Interpersonal } \\
\text { difficulties } \\
\text { Suicidality } \\
\text { Minimisation } \\
\text { Lack of help-seeking }\end{array}$ \\
\hline $\begin{array}{l}\text { Le Blanc-Louvry, } \\
\text { Papin, Vaz, \& Proust } \\
\text { (2013) }\end{array}$ & France & $\begin{array}{l}\text { Case reports } \\
\text { (hospital) }\end{array}$ & $\begin{array}{l}\mathrm{N}=3 \text {, only } 1 \text { IPV- } \\
\text { related } \\
29 \text { year old woman } \\
\text { strangled twice within } \\
\text { a few minutes by } \\
\text { spouse. First attempt } \\
\text { manual, front on; } \\
\text { second was } \\
\text { 'chokehold' from } \\
\text { behind. }\end{array}$ & $\begin{array}{l}\text { JBI Case Reports: } \\
5 / 8 \\
\text { Nil for } 3 / 4 / 6\end{array}$ & $\begin{array}{l}\text { Brain CT: wide temporal } \\
\text { hypodensity in left middle } \\
\text { cerebral artery area } \\
\text { Doppler: left carotid artery } \\
\text { thrombosis due to major } \\
\text { dissection }\end{array}$ & $\begin{array}{l}\text { Dysesthesia right hand and foot } \\
\text { Headache } \\
\text { Facial paralysis } \\
\text { Right-side hemiplegia } \\
\text { Broca-like aphasia } \\
\text { Lateral homonymous hemianopia } \\
\\
\text { Symptoms all persistent and worsening after } \\
3 \text { weeks; discharged to neurorehabilitation, } \\
\text { further deteriorated with seizures }\end{array}$ & $\begin{array}{l}\text { Pathological: Arterial } \\
\text { dissection and } \\
\text { thrombosis } \\
\text { Neurological: } \\
\text { Aphasia } \\
\text { Headache }\end{array}$ \\
\hline Malek et al. (2000) & USA & $\begin{array}{l}\text { Case reports } \\
\text { (hospital) }\end{array}$ & $\begin{array}{l}\mathrm{N}=3 \\
\text { Age } 24-43 \text {, all } \\
\text { victims of } \\
\text { strangulation by } \\
\text { partner }\end{array}$ & $\begin{array}{l}\text { JBI Case Reports: } \\
7 / 8 \\
\text { Nil for } 6\end{array}$ & $\begin{array}{ll}\text { 1. } & \begin{array}{l}\text { Left posterior } \\
\text { frontal lobe stroke, } \\
\text { delayed by } 3\end{array} \\
& \text { months } \\
\text { 2. } & \text { Right opercular } \\
\text { stroke, delayed by } \\
\text { 3. } & \text { 3 months } \\
\text { Bilateral frontal } \\
\text { infarcts in } \\
\text { watershed } \\
\text { distribution of } \\
\text { MCA \& ACA. }\end{array}$ & $\begin{array}{ll}\text { 1. } & \begin{array}{l}\text { Dysarthria; Residual right hand } \\
\text { and digit weakness and } \\
\text { numbness; no symptoms at } 8\end{array} \\
\text { month follow-up } \\
\text { 2. }\end{array}$ & $\begin{array}{l}\text { Pathological: Stroke } \\
\text { Bilateral, symmetrical, } \\
\text { high cervical CA } \\
\text { dissection } \\
\text { NB all cases had } \\
\text { hyperthyroidism } \\
\text { described as 'coexisting } \\
\text { medical problem'. } \\
\text { However, the thyroid } \\
\text { can be damaged by } \\
\text { strangulation. } \\
\text { Neurological: } \\
\text { Dysarthria } \\
\text { Dysphasia }\end{array}$ \\
\hline Meel (2015) & South Africa & $\begin{array}{l}\text { Case report } \\
\text { (hospital) }\end{array}$ & $\begin{array}{l}\mathrm{N}=1 \\
23 \text { year old woman } \\
\text { sexually assaulted by } \\
\text { colleague }\end{array}$ & $\begin{array}{l}\text { JBI Case Reports: } \\
5 / 8 \\
\text { Nil for } 4 / 5 / 6\end{array}$ & $\begin{array}{l}\text { Symptoms of venous } \\
\text { congestion: bilateral sub- } \\
\text { conjunctival haemorrhage } \\
\text { CT neck-only: hyoid fracture }\end{array}$ & $\begin{array}{l}\text { Loss of consciousness (GCS 3/15) } \\
\text { Seizures, suggesting cerebral hypoxia } \\
\text { Discharged after a week "with no } \\
\text { neurological deficits" } \\
\text { No follow-up }\end{array}$ & $\begin{array}{l}\text { Pathological: } \\
\text { Venous congestion } \\
\text { Hyoid fracture }\end{array}$ \\
\hline
\end{tabular}




\begin{tabular}{|c|c|c|c|c|c|c|c|}
\hline Reference & Country & Study Design & Sample & Quality & Neuropathology & $\begin{array}{l}\text { Neuropsychological outcomes } \\
\text { (neurological, cognitive, emotional, } \\
\text { behavioural) }\end{array}$ & $\begin{array}{l}\text { Additional to hypoxic- } \\
\text { ischaemic? }\end{array}$ \\
\hline $\begin{array}{l}\text { Messing, Patch, Wilson, } \\
\text { Kelen, \& Campbell } \\
\text { (2018) }\end{array}$ & USA & $\begin{array}{l}\text { Analytical cross- } \\
\text { sectional }\end{array}$ & $\begin{array}{l}\mathrm{N}=1,008 \\
\text { Community-living } \\
\text { women recruited } \\
\text { through contact with } \\
\text { police for IPV; mean } \\
\text { age 32; } \\
803(80 \%) \text { reported } \\
\text { strangulation; } 38 \% \\
\text { multiple strangulation } \\
\text { attempts }\end{array}$ & $\begin{array}{l}\text { JBI for Cross Sectional: } \\
8 / 8\end{array}$ & & $\begin{array}{l}\text { More likely than non-strangled IPV } \\
\text { survivors to: } \\
\text { - believe partner capable of killing them } \\
\text { (ARR, 1.81) } \\
\text { - have suffered miscarriage due to abuse } \\
\text { (ARR, 2.95) } \\
\text { - have lost consciousness for over an hour } \\
\text { (ARR, 5.08) } \\
\text { - feel powerless (ARR, 2.62) } \\
27 \% \text { reported loss of consciousness } \\
\text { Multiple strangulation significantly } \\
\text { associated with miscarriage }\end{array}$ & $\begin{array}{l}\text { Psychological: } \\
\text { Existential fear } \\
\text { Powerlessness } \\
\text { Other: } \\
\text { Miscarriage }\end{array}$ \\
\hline $\begin{array}{l}\text { Messing, Thaller, \& } \\
\text { Bagwell (2014) }\end{array}$ & USA & $\begin{array}{l}\text { Analytical cross- } \\
\text { sectional }\end{array}$ & $\begin{array}{l}\mathrm{N}=432 \\
\text { Women recruited at } \\
\text { scene of police- } \\
\text { involved IPSV } \\
\text { incidents; age range } \\
18-62 \\
315(73 \%) \text { reported } \\
\text { strangulation }\end{array}$ & $\begin{array}{l}\text { JBI Cross Sectional: } \\
7 / 8 \\
\text { Nil for } 6\end{array}$ & & $\begin{array}{l}\text { Strangulation significantly associated with } \\
\text { sexual abuse (OR, 2.74) and rape (OR, 2.19) } \\
\text { Women experiencing forced sex more likely } \\
\text { to experience strangulation, PTSD, and } \\
\text { shame (direction of interactions not } \\
\text { analysed) }\end{array}$ & $\begin{array}{l}\text { Psychological: } \\
\text { PTSD } \\
\text { Shame }\end{array}$ \\
\hline Miao et al. (2008) & China & $\begin{array}{l}\text { Case report } \\
\text { (hospital) }\end{array}$ & $\begin{array}{l}\mathrm{N}=1 \\
21 \text { year old woman } \\
\text { strangled in IPV }\end{array}$ & $\begin{array}{l}\text { JBI Case Reports: } \\
4 / 8 \\
\text { Nil for } 3 / 4 / 6 / 7\end{array}$ & Bilateral basal ganglia lesions & $\begin{array}{l}\text { No respiration for } 3 \text { minutes but heart } \\
\text { continued with CPR } \\
\text { Unconscious for } 24 \text { hours } \\
\text { No symptoms } \\
5 \text { days later, resting left hand tremor and } \\
\text { mild dysarthria. After } 8 \text { days, bradykinesia, } \\
\text { severe tremor, rigidity of all extremities; } \\
\text { delayed Parkinsonism. Treated with } \\
\text { hyperbaric oxygen and asymptomatic at } 12 \\
\text { months }\end{array}$ & $\begin{array}{l}\text { Neurological: } \\
\text { Dysarthria }\end{array}$ \\
\hline
\end{tabular}




\begin{tabular}{|c|c|c|c|c|c|c|c|}
\hline Reference & Country & Study Design & Sample & Quality & Neuropathology & $\begin{array}{l}\text { Neuropsychological outcomes } \\
\text { (neurological, cognitive, emotional, } \\
\text { behavioural) }\end{array}$ & $\begin{array}{l}\text { Additional to hypoxic- } \\
\text { ischaemic? }\end{array}$ \\
\hline $\begin{array}{l}\text { Milligan \& Anderson } \\
(1980)\end{array}$ & UK & $\begin{array}{l}\text { Case reports } \\
\text { (hospital) }\end{array}$ & $\begin{array}{l}\mathrm{N}=2 \\
\text { Women strangled by } \\
\text { husbands }(37 \& 28)\end{array}$ & $\begin{array}{l}\text { JBI Case Reports: } \\
7 / 8 \\
\text { Nil for } 6\end{array}$ & $\begin{array}{l}\text { Partial occlusion of left } \\
\text { carotid artery } 1.5 \mathrm{~cm} \text { distal to } \\
\text { bifurcation. } \\
\text { Diagnosis: stroke due to ICA } \\
\text { lesion } \\
\text { Large infarct left fronto- } \\
\text { parietal. Tapering occlusion } \\
\text { of internal carotid } 2 \mathrm{~cm} \text { distal } \\
\text { to bifurcation. } \\
\text { Diagnosis: stroke due to ICA } \\
\text { lesion }\end{array}$ & $\begin{array}{l}3 \text { days following strangulation: } \\
\text { Confusion } \\
\text { Headache } \\
\text { Right-sided weakness and sensory loss } \\
\text { Dysphasia } \\
\text { Ptosis, then Horner's syndrome } \\
\text { Hyperreflexia } \\
8 \text { year GP review asymptomatic } \\
\\
\text { Day after strangulation: } \\
\text { Collapsed but conscious } \\
\text { Severe dysphasia } \\
\text { Right hemianopia } \\
\text { Right hemiplegia } \\
\text { Right sensory deficit } \\
2 \text { year GP review, speech hesitancy }\end{array}$ & $\begin{array}{l}\text { Pathological: } \\
\text { Arterial lesion and } \\
\text { stroke } \\
\text { Neurological: } \\
\text { Headache } \\
\text { Dysphasia and speech } \\
\text { hesitancy }\end{array}$ \\
\hline Mittal et al. (2018) & USA & $\begin{array}{l}\text { Analytical cross- } \\
\text { sectional using } \\
\text { secondary data } \\
\text { (from RCT) }\end{array}$ & $\begin{array}{l}\mathrm{N}=175 \\
\text { Participants in trial } \\
\text { for HIV-IPV } \\
\text { prevention } \\
\text { intervention for } \\
\text { abused women } \\
\text { Majority }(n=103) \\
\text { reported } \\
\text { strangulation. Age } \\
\text { range } 18-49 ; 41 \% \\
\text { African American. }\end{array}$ & $\begin{array}{l}\text { JBI Cross Sectional: } \\
8 / 8\end{array}$ & & $\begin{array}{l}\text { Strangulation significantly correlated with } \\
\text { depression } \\
\text { In logistic regression, significant factor (OR } \\
=2.40,95 \% \text { CI } 1.29-4.50)\end{array}$ & $\begin{array}{l}\text { Psychological: } \\
\text { Depression }\end{array}$ \\
\hline $\begin{array}{l}\text { Plattner, Bolliger, \& } \\
\text { Zollinger (2004) }\end{array}$ & Switzerland & $\begin{array}{l}\text { Retrospective, } \\
\text { cross sectional } \\
\text { analysis of } \\
\text { clinical } \\
\text { examination data }\end{array}$ & $\begin{array}{l}\mathrm{N}=134 \\
\text { Hospital records of } \\
\text { survived } \\
\text { strangulation cases } \\
114(85 \%) \text { victims } \\
\text { female. All male } \\
\text { victims assaulted by } \\
\text { men. In } 47 \text { (35\%) } \\
\text { cases strangulation } \\
\text { inflicted during rape. } \\
4 / 134 \text { were strangled } \\
\text { from behind ("carotid } \\
\text { sleeper") with severe } \\
\text { effects. }\end{array}$ & $\begin{array}{l}\text { JBI Cross sectional: } \\
5 / 8 \\
\text { Nil for } 6 / 7 / 8\end{array}$ & $\begin{array}{l}21 \% \text { had petechial } \\
\text { haemorrhages }\end{array}$ & $\begin{array}{l}20 \% \text { dyspnoea } \\
10 \% \text { "nearly" fainted } \\
11 \% \text { lost consciousness } \\
3 \% \text { incontinent }\end{array}$ & $\begin{array}{l}\text { Pathological: } \\
\text { Petechiae } \\
\text { Neurological: } \\
\text { Incontinence }\end{array}$ \\
\hline
\end{tabular}




\begin{tabular}{|c|c|c|c|c|c|c|c|}
\hline Reference & Country & Study Design & Sample & Quality & Neuropathology & $\begin{array}{l}\text { Neuropsychological outcomes } \\
\text { (neurological, cognitive, emotional, } \\
\text { behavioural) }\end{array}$ & $\begin{array}{l}\text { Additional to hypoxic- } \\
\text { ischaemic? }\end{array}$ \\
\hline $\begin{array}{l}\text { Pritchard, Reckdenwald, } \\
\text { Nordham, \& Holton } \\
\text { (2018) }\end{array}$ & USA & $\begin{array}{l}\text { Retrospective, } \\
\text { cross sectional } \\
\text { analysis of police } \\
\text { records }\end{array}$ & $\begin{array}{l}\mathrm{N}=591 \text { cases } \\
\text { charged as IPV } \\
68 \text { explicit } \\
\text { strangulation cases } \\
\text { and } 101 \text { possible } \\
\text { cases. } 13 \% \text { choke } \\
\text { hold, } 6 \% \text { pinned } \\
\text { against wall/floor. }\end{array}$ & $\begin{array}{l}\text { JBI Cross sectional: } \\
5 / 8 \\
\text { Nil for } 6 / 7 / 8\end{array}$ & $16 \%$ petechiae & $\begin{array}{l}22 \% \text { cognitive symptoms (e.g. memory } \\
\text { problems, slurred speech) } \\
2 \% \text { dysphagia } \\
2 \% \text { voice changes } \\
25 \% \text { breathing difficulties }\end{array}$ & $\begin{array}{l}\text { Neurological: } \\
\text { Petechiae }\end{array}$ \\
\hline Purvin (1997) & USA & $\begin{array}{l}\text { Case report } \\
\text { (hospital) }\end{array}$ & $\begin{array}{l}\mathrm{N}=1 \\
30 \text { year old woman } \\
\text { strangled by } \\
\text { boyfriend }\end{array}$ & $\begin{array}{l}\text { JBI Case Reports: } \\
7 / 8 \\
\text { Nil for } 6\end{array}$ & $\begin{array}{l}\text { Carotid artery dissection with } \\
\text { pseudoaneurysm formation } \\
\text { and high-grade narrowing }\end{array}$ & $\begin{array}{l}\text { Severe left-sided headache } \\
\text { Followed after two weeks by left-sided post- } \\
\text { glandionic Horner's Syndrome }\end{array}$ & $\begin{array}{l}\text { Pathological: } \\
\text { CA dissection }\end{array}$ \\
\hline $\begin{array}{l}\text { Ralston, Rable, Larson, } \\
\text { Handmaker, \& Lifshitz } \\
\text { (2019) }\end{array}$ & USA & $\begin{array}{l}\text { Retrospective } \\
\text { analysis of } \\
\text { symptom self- } \\
\text { report in forensic } \\
\text { nursing records }\end{array}$ & $\begin{array}{l}\mathrm{N}=19 \\
\text { Patients presenting } \\
\text { with IPV injuries } \\
17 / 19 \text { female; mean } \\
\text { age } 32 \\
100 \% \text { reported } \\
\text { strangulation } \\
\text { Excluded those who } \\
\text { had also been } \\
\text { sexually assaulted }\end{array}$ & $\begin{array}{l}\text { JBI Cross sectional: } \\
6 / 8 \\
\text { Nil for } 6 / 7\end{array}$ & $21.1 \%$ petechiae & $\begin{array}{l}84 \% \text { dizziness } \\
79 \% \text { headache } \\
79 \% \text { breathing difficulty } \\
68 \% \text { pain } \\
16 \% \text { nausea and vomiting } \\
26 \% \text { loss of consciousness } \\
37 \% \text { confusion and lack of orientation } \\
11 \% \text { sleepiness } \\
58 \% \text { visual changes } \\
5 \% \text { incontinence } \\
58 \% \text { voice changes } \\
11 \% \text { loss of hearing }\end{array}$ & $\begin{array}{l}\text { Pathological: } \\
\text { Petechiae } \\
\text { Neurological: } \\
\text { Pain } \\
\text { Incontinence } \\
\text { Breathing difficulty }\end{array}$ \\
\hline $\begin{array}{l}\text { Shields, Corey, } \\
\text { Weakley-Jones, \& } \\
\text { Stewart (2010) }\end{array}$ & USA & $\begin{array}{l}\text { Retrospective } \\
\text { analysis of } \\
\text { medicolegal } \\
\text { evaluations } \\
\text { records }\end{array}$ & $\begin{array}{l}\mathrm{N}=102 \\
101 / 102 \text { female } \\
79 \% \text { strangled by } \\
\text { intimate partner, } 7 \% \\
\text { by stranger; } 9 \% \\
\text { pregnant at time of } \\
\text { attack. Age 17-68 (M } \\
=31 \text { ). 30\% African } \\
\text { American. }\end{array}$ & $\begin{array}{l}\text { JBI Cross Sectional: } \\
6 / 8 \\
\text { Nil for } 6 / 7\end{array}$ & $\begin{array}{l}\text { Petechiae } \\
\text { Stroke } \\
\text { Right vertebral artery } \\
\text { dissection } \\
\text { Left cerebral infarction of } \\
\text { distal posterior cerebral artery }\end{array}$ & $\begin{array}{l}38 \% \text { loss of consciousness } \\
\text { Miscarriage } \\
\text { Incontinence } \\
\text { Coma } \\
\text { Difficulty breathing } \\
\text { Dysphagia } \\
\text { Dyspnoea } \\
\text { Dizziness } \\
\text { Amnesia for event } \\
\text { Minimisation/lying about event due to fear, } \\
\text { shame, embarrassment } \\
\text { Experienced verbal threats to kill } \\
\text { "He's going to kill me if not the baby" (p. } \\
\text { 321) }\end{array}$ & $\begin{array}{l}\text { Pathological: } \\
\text { Petechiae } \\
\text { Stroke } \\
\text { Vertebral artery } \\
\text { dissection } \\
\text { Neurological: } \\
\text { Pain } \\
\text { Incontinence } \\
\text { Breathing difficulty } \\
\text { Psychological: }\end{array}$ \\
\hline
\end{tabular}




\begin{tabular}{|c|c|c|c|c|}
\hline $\begin{array}{l}\text { Smith, Mills, \& } \\
\text { Taliaferro (2001) }\end{array}$ & USA & $\begin{array}{l}\text { Cross-sectional } \\
\text { survey-based } \\
\text { study }\end{array}$ & $\begin{array}{l}\mathrm{N}=101 \\
\text { Recruited from } \\
\text { women's refuges and } \\
\text { ED patients including } \\
57 / 102 \text { strangled } \\
\text { twice or more }\end{array}$ & $\begin{array}{l}\text { JBI Cross Sectional } \\
\text { Studies } \\
4 / 8 \\
\text { Nil for } 2 / 5 / 6 / 7\end{array}$ \\
\hline
\end{tabular}

"I could have killed you. You're lucky I didn't" (p. 323)

Pain

Minimisation/lying

about event due to fear

shame, embarrassment

Existential threat

Miscarriage

Multiple strangulation vs one incident Neurological:

increases risk of $(*$ for significant

difference): $\quad$ Tinnitus

Personality change

Depression, suicidality

Nightmares*, insomnia

Anxiety

Diagnosed PTSD

Dizziness/light-headedness*

Pain*, headache

Memory loss*

Vision changes

Tinnitus*

Ptosis

Weakness*

Facial droop

Paralysis

Paralysis

Loss of sensation

Muscle spasms

Voice changes*

Multiple strangulation subjects more

frequently report neurological and

psychological symptoms than single attack

subjects
Strack, McClane, \&

Hawley (2001)
USA

\begin{tabular}{|c|c|c|}
\hline \multirow{4}{*}{$\begin{array}{l}\text { Cross sectional } \\
\text { analysis of } \\
\text { prosecution files } \\
\text { for domestic } \\
\text { violence }\end{array}$} & $\begin{array}{l}\mathrm{N}=300 \text { strangulation } \\
\text { victims }\end{array}$ & $\begin{array}{l}\text { JBI Cross Sectional } \\
\text { Studies }\end{array}$ \\
\hline & $99 \%$ female & \\
\hline & Only two were & $6 / 8$ \\
\hline & $\begin{array}{l}\text { male victim. Mean } \\
\text { age } 32.10 \text { were } \\
\text { pregnant at time of } \\
\text { attack. }\end{array}$ & Nil for $6 / 7$ \\
\hline
\end{tabular}

Delayed presentation - only
$39 \%$ had symptoms on day of
incident
Only $15 \%$ had symptoms
sufficiently visible to be

sufficiently visible to be

photographed for evidence

Petechiae

Bilateral sub-conjunctiva

haemorrhage
Pain

Amnesia

Ataxia

Uncontrollable shaking

Defecation

Hyperventilation

Light-headness

Visual changes ("black and white" or "black

spots", p. 305)

Loss of consciousness

Headache

Nausea and vomiting

Vomiting blood

Dysphagia
Neurological:

Uncontrollable shaking

Hyperventilation

Voice changes

Delayed presentation

Psychological:

Existential threat

Miscarriage 
Miscarriage within 24h

\begin{tabular}{|c|c|c|c|c|c|c|c|}
\hline $\begin{array}{l}\text { Thomas, Joshi, \& } \\
\text { Sorenson (2014) }\end{array}$ & USA & Qualitative & $\begin{array}{l}\mathrm{N}=17 \\
\text { female shelter } \\
\text { residents who had } \\
\text { experienced } \\
\text { strangulation, aged } \\
21-47,14 / 17 \text { African } \\
\text { American, 13/17 } \\
\text { experienced multiple } \\
\text { strangulation, } 4 / 17 \\
\text { pregnant }\end{array}$ & $\begin{array}{l}\text { JBI Qualitative Research } \\
9 / 10 \\
\text { Nil for } 7\end{array}$ & & $\begin{array}{l}\text { Shock } \\
\text { Intense sense of vulnerability } \\
\text { Powerlessness } \\
\text { Pain } \\
\text { Loss of consciousness } \\
\text { Experienced death threats } \\
\text { Belief in imminent death (16/17) } \\
\text { "I thought I was going to die, I really did, } \\
\text { because I got real clammy and everything } \\
\text { just got real dark" (p. 130) } \\
\text { "painful to watch the man who so-called } \\
\text { loves you try to kill you" (p. 130) } \\
\text { Immediate and lasting fear } \\
\text { Subsequent compliant/submissive behaviour } \\
\text { Aggression towards perpetrator } \\
\text { Not leaving house }\end{array}$ & $\begin{array}{l}\text { Psychological: } \\
\text { Shock } \\
\text { Vulnerability } \\
\text { Existential fear } \\
\text { Powerlessness } \\
\text { Emotional pain } \\
\\
\text { Behaviour: } \\
\text { Compliant } \\
\text { Submissive } \\
\text { Aggressive } \\
\text { Not leaving house }\end{array}$ \\
\hline Wilbur et al. (2001) & USA & $\begin{array}{l}\text { Analytical cross } \\
\text { sectional (survey- } \\
\text { based) }\end{array}$ & $\begin{array}{l}\mathrm{N}=62 \\
\text { Women's shelters and } \\
\text { hospital-based } \\
\text { violence prevention } \\
\text { centre } \\
42(68 \%) \text { had been } \\
\text { strangled by partner. } \\
42 \% \text { Hispanic and } \\
16 \% \text { African } \\
\text { American. Average } \\
\text { length of abuse } \\
\text { before strangulation } 3 \\
\text { years. }\end{array}$ & $\begin{array}{l}\text { JBI Cross Sectional } \\
\text { Studies } \\
6 / 8 \\
\text { Nil for } 6 / 7\end{array}$ & $54 \%$ petechiae & $\begin{array}{l}61 \% \text { dizziness } \\
17 \% \text { loss of consciousness } \\
28 \% \text { visual changes } \\
36 \% \text { tinnitus } \\
45 \% \text { voice changes } \\
44 \% \text { dysphagia } \\
68 \% \text { pain } \\
85 \% \text { breathing difficulties } \\
11 \% \text { miscarriage } \\
11 \% \text { incontinence } \\
20 \% \text { ptosis } \\
10 \% \text { facial droop } \\
23 \% \text { weakness/paralysis } \\
31 \% \text { loss of sensation } \\
31 \% \text { amnesia } \\
81 \% \text { depression } \\
31 \% \text { suicidality } \\
67 \% \text { insomnia } \\
70 \% \text { nightmares } \\
83 \% \text { anxiety } \\
\text { PTSD (no figure given) }\end{array}$ & $\begin{array}{l}\text { Pathological: } \\
\text { Petechiae } \\
\text { Neurological: } \\
\text { Tinnitus } \\
\text { Voice change } \\
\text { Pain } \\
\text { Breathing difficulties } \\
\text { Incontinence } \\
\text { Psychological: } \\
\text { Depression } \\
\text { Suicidality } \\
\text { Insomnia } \\
\text { Nightmares } \\
\text { Anxiety } \\
\text { PTSD } \\
\text { Miscarriage }\end{array}$ \\
\hline
\end{tabular}




\begin{tabular}{|c|c|c|c|c|c|c|c|}
\hline Reference & Country & Study Design & Sample & Quality & Neuropathology & $\begin{array}{l}\text { Neuropsychological outcomes } \\
\text { (neurological, cognitive, emotional, } \\
\text { behavioural) }\end{array}$ & $\begin{array}{l}\text { Additional to hypoxic- } \\
\text { ischaemic? }\end{array}$ \\
\hline Yen et al. (2007) & $\begin{array}{l}\text { Austria, } \\
\text { Switzerland }\end{array}$ & $\begin{array}{l}\text { Case reports from } \\
\text { forensic } \\
\text { examination } \\
\text { (using existing } \\
\text { radiological } \\
\text { findings) }\end{array}$ & $\begin{array}{l}\mathrm{N}=14 \\
2 \text { men, } 12 \text { women } \\
\text { Mean age } 33\end{array}$ & $\begin{array}{l}\text { JBI Case Reports: } \\
6 / 8 \\
\text { Nil for } 6 / 7\end{array}$ & $\begin{array}{l}\text { Petechiae } \\
\text { Extensive infarction of both } \\
\text { cerebellar hemispheres } \\
\text { Haemorrhage of lymph nodes } \\
\text { Patients reported significant, } \\
\text { understandable distress } \\
\text { having to wear neck coil for } \\
\text { MRI, which may have caused } \\
\text { motion artefacts and reduced } \\
\text { quality of diagnostics }\end{array}$ & $\begin{array}{l}\text { Loss of consciousness/"blackout" } \\
\text { Incontinence } \\
\text { Dizziness } \\
\text { Pain } \\
\text { Hallucinations before losing consciousness } \\
\text { Impaired vision: seeing "black void" (p.116) } \\
\text { reported by } 50 \% \\
\text { Anxiety }\end{array}$ & $\begin{array}{l}\text { Pathological: } \\
\text { Cerebellar infarction } \\
\text { Petechiae } \\
\text { Haemorrhage of lymph } \\
\text { nodes } \\
\text { Neurological: } \\
\text { Incontinence } \\
\text { Pain } \\
\text { "Black void" in vision } \\
\text { Psychological: } \\
\text { Hallucinations } \\
\text { Anxiety }\end{array}$ \\
\hline Zilkens et al. (2016) & Australia & $\begin{array}{l}\text { Analytical cross- } \\
\text { sectional study } \\
\text { using existing } \\
\text { routine } \\
\text { admissions data }\end{array}$ & $\begin{array}{l}\mathrm{N}=1,064 \text { women } \\
\text { referred to a sexual } \\
\text { assault centre, } 79 \text { of } \\
\text { whom had been } \\
\text { strangled. Mean age } \\
29.5 \% \text { were } \\
\text { pregnant. Odds of } \\
\text { strangulation were } \\
8.4 \text { times more likely } \\
\text { for someone sexually } \\
\text { assaulted by intimate } \\
\text { partner vs stranger. }\end{array}$ & $\begin{array}{l}\text { JBI Cross Sectional } \\
\text { Studies } \\
6 / 8 \\
\text { Nil for } 6 / 7\end{array}$ & $\begin{array}{l}27.8 \% \text { petechiae } \\
3.8 \% \text { subconjunctival } \\
\text { haemorrhage }\end{array}$ & $\begin{array}{l}46.8 \% \text { pain } \\
34.2 \% \text { dysphagia } \\
15.2 \% \text { voice changes } \\
8.9 \% \text { breathlessness } \\
8.9 \% \text { loss of consciousness } \\
8.9 \% \text { dizziness } \\
2.5 \% \text { blurred vision } \\
1.3 \% \text { incontinence } \\
\text { Deprivation of liberty \& verbal threats more } \\
\text { likely than in non-strangulation assaults }\end{array}$ & $\begin{array}{l}\text { Pathological: } \\
\text { Petechiae } \\
\text { Neurological: } \\
\text { Breathlessness } \\
\text { Incontinence } \\
\text { Pain } \\
\text { Psychological: } \\
\text { Impact of deprivation } \\
\text { of liberty and verbal } \\
\text { threats }\end{array}$ \\
\hline
\end{tabular}




\section{Summary of main findings}

This review's main objective was to ascertain what evidence we have for the neurological, cognitive, behavioural, and psychological impact of strangulation within IPV and sexual assault.

\section{Neurological outcomes}

21/27 studies reported potentially serious neurological outcomes. Loss of, and alterations to, consciousness, were widespread, suggesting at least mild brain injury per the Mayo classification system (Malek et al., 2007). For those studies reporting incidence, loss of consciousness ranged from $8.9 \%$ (Zilkens et al., 2016) to $38 \%$ of strangulation attempts (Shields et al., 2010). Importantly, figures were higher when taken from medical settings, where consciousness was assessed objectively. When women self-reported at a later date, lower figures may be due to amnesia, or not understanding what 'blacking out' or 'passing out' signify. Other widely reported neurological consequences, with number of articles in parentheses, include: changes to vision, including hemianopia (9); pain (9); dysarthria, dysphonia, and other voice changes (9); headache (8); dyspnoea or breathing difficulty (7); facial or limb paralysis (7); dysthesia or loss of or changes to sensation (7); swallowing difficulty or dysphagia (6); lightheaded or dizzy (6); urinary or faecal incontinence (6); limb weakness (6); tinnitus (4); dysphasia (4); spasms/tremor/shaking (3); seizures (2); confusion (2); nausea and vomiting (2); ptosis and Horner's Syndrome (2); coma (2); ataxia (2); facial droop (2); hyperreflexia (1). In the eight studies that reported follow-up outcomes, or were based on surveys or interviews with survivors at a later date, ongoing symptoms, with number of articles in parentheses, included: pain, tinnitus, vision changes, paralysis (4); headache, sensory and voice changes (3); swallowing difficulty, ptosis, incontinence, facial droop (2); seizures, dizziness, breathing difficulty, and muscle spasm (1). 


\section{Cognitive outcomes}

Only one case report mentioned an immediate cognitive outcome, which was amnesia for the strangulation incident (De Boos, 2019). Five studies reported delayed cognitive outcomes, all of which cited amnesia. Farr (2002) reported two victims having been taken into public by the attacker afterwards and making no attempt to escape, which is deemed to be "traumatic immobility" (p. 276). This may have had a psychological basis - fear - but could also be cognitive, e.g. agnosia or lack of initiation. Pritchard et al. (2018) reported 22\% of police records mentioning "any psychological symptoms", but then refers to "memory problems, slurred speech etc" (p. 171), which seem to be more neurocognitive, and are, unfortunately, not separated out.

\section{Psychological outcomes}

Only four studies reported on psychological distress in the immediate aftermath of the strangulation, which all hinged on a sense of existential threat, the firm conviction that they were about to die (De Boos, 2019; Funk \& Schuppel, 2003; Shields et al., 2010; Strack et al., 2001). A further 13 studies reported on delayed psychological outcomes. These included (with number of articles in parentheses): existential threat (7); depression, anxiety, suicidality, and nightmares (4); insomnia, PTSD (3); generalised fear and feelings of danger, powerlessness and vulnerability, dissociation at the time of the attack, and ongoing, including possible dissociative seizures, minimisation and denial of events (2); and then single articles reported increased shame, hypervigilance, participants believing they had actually died, interpersonal difficulties, personality change, feelings of worthlessness, further unspecified trauma reaction, and exacerbation of existing mental health difficulties.

The verbal threats to kill made by attackers were mentioned in three studies, and give substance to the existential fear reported by victims (Shields et al., 2010, Strack et al., 2001; 
Zilkens et al., 2016). Chilling examples include: "I am going to commit an OJ on you and leave no visible marks" or "Die, die" (Strack et al., 2001, p. 307). Messing et al. (2018) found that IPV victims who were strangled had significantly increased odds of believing their partner could and would kill them (ARR, 1.81). Both studies by Joshi et al (2014 \& 2018) reported dissociative reactions at the time of the strangulation, seeing life flashing before their eyes, and possible non-epileptic absence seizures as a long-term consequence: "I black out, you can be talking to me right now and I can't see you, can't hear you (p. 1,636). One of Farr's participants (2002) describes the sense of "being killed and watching it" (p. 275). In Yen et al. (2007) 7/14 of forensic examinations contain reports of seeing a "black void". Thomas et al. (2014) draw attention to the highly personal nature of the crime, as well as this dissociative element, with a victim stating it is "painful to watch the man who so-called loves you try to kill you" (p. 130)

An incidental, but sad finding in several of the studies was miscarriage following the strangulation event. Messing et al. (2018) reported increased odds (ARR, 2.95) for strangled versus non-strangled IPV survivors, and that this increased for those who had experienced multiple incidents. Wilbur and colleagues (2001) reported an $11 \%$ incidence amongst women in refuges who had been strangled. Although miscarriage does not directly fall within immediate psychological outcomes, the potential effect does, as large effects have been shown elsewhere for depression, guilt, and complicated grief (Adolfsson, 2011).

\section{Behavioural outcomes}

One qualitative study (Thomas et al., 2014) mentioned behavioural changes after strangulation, indicative of power dynamics. These included increased compliant and submissive behaviour, self-isolation and not leaving the house, and, for one participant, increased aggression towards her partner. Another qualitative study discussed the survivalist 
mode the victim entered following the realisation that death was imminent: "then I knew it's either him or me" (Eiskovits \& Winstok, 2002, p. 695). Several studies drew attention to the lack of help-seeking behaviour by strangulation victims (Joshi et al, 2012; Pritchard et al., 2018; Ralston et al., 2019). Only 39\% of women at a refuge who had been strangled had sought medical attention, despite more than half having been strangled twice or more (Smith et al., 2001). This dropped to 5\% in a study of 300 prosecution files (Strack et al., 2001).

\section{Is there a distinction between strangulation and hypoxic-ischaemic outcomes?}

Our second review question was the extent to which these reported sequelae differed from typical symptoms of other hypoxic-ischaemic injuries such as cardiac arrest, given the similar mechanisms, not least that the sinus reflex can be triggered in strangulation. Table 2 compares outcomes, using the International Brain Injury Association's delineation of neurological and neurobehavioural outcomes (Arciniegas, 2012), compared to the findings from our literature review.

Table 2. Neuropsychological outcomes of hypoxic-ischaemic injury versus strangulation

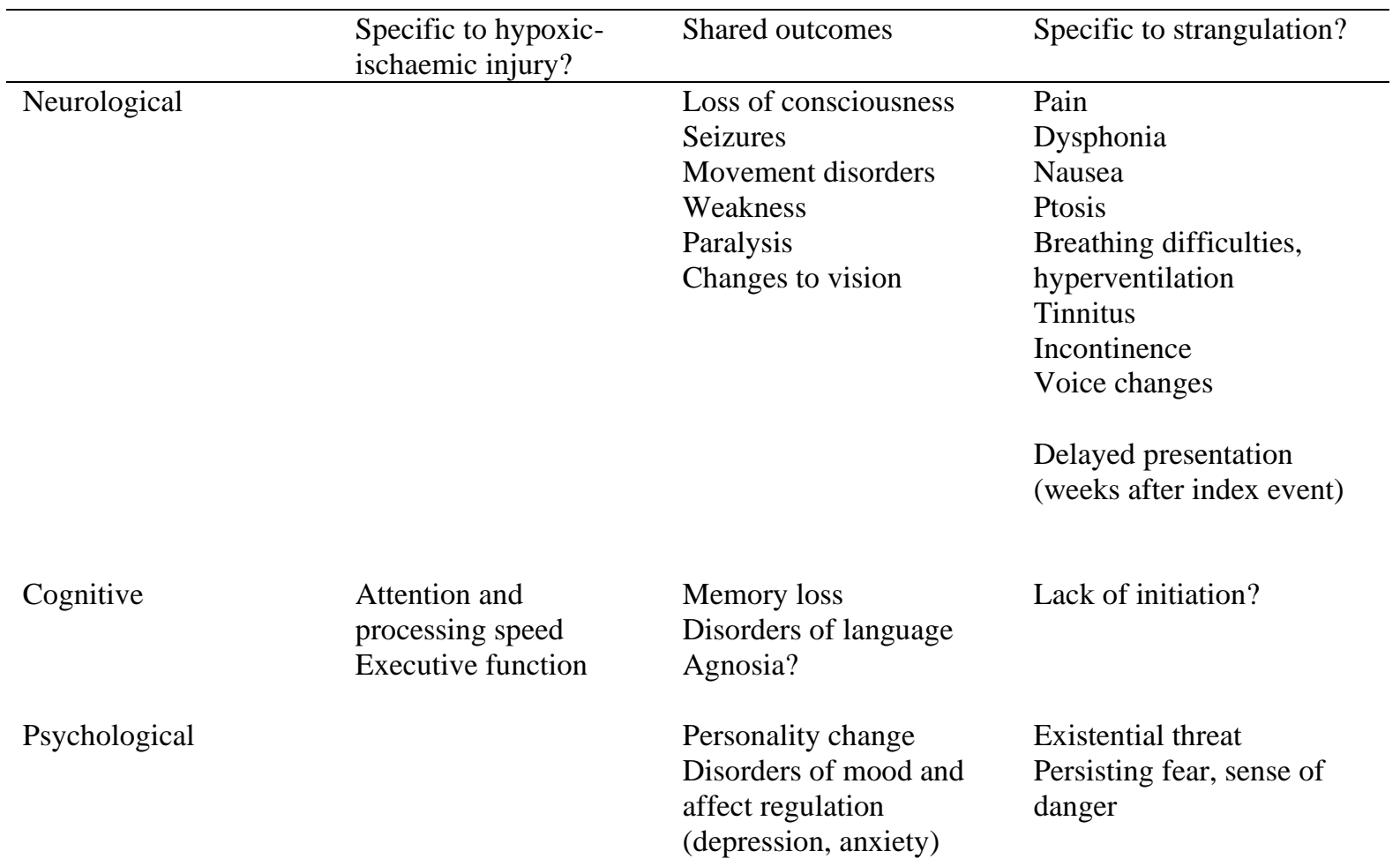


Sense of vulnerability and powerlessness

Dissociation

Hypervigilance

Suicidality

PTSD

Trauma reaction

Nightmares

Insomnia

Exacerbation of existing mental health difficulties Interpersonal difficulties Minimisation and denial

Hallucinations

Shame

Impact of verbal threats

and deprivation of liberty

Indirect effect of

miscarriage

Behavioural

Behavioural disturbance, including aggression

Compliant and submissive behaviour Self-isolating

Lack of help-seeking

The information in Table 2 is clearly limited by the data reported in the studies available for our review. Some strangulation events result in complete occlusion of both air and blood flow, can trigger the carotid sinus reflex, and would therefore include all hypoxic-ischaemic outcomes, by definition. Given the lack of reporting on cognitive outcomes in the studies we reviewed, we cannot state that strangulation does not lead to difficulties with attention, speed of processing, or executive function. In fact, several case reports outlined frontal lobe and basal ganglia damage (Malek et al., 2000; Miao et al., 2008; Milligan \& Anderson, 1980). Unfortunately, these studies were limited to anatomical changes. Similarly, some of the neurological outcomes reported in the strangulation literature are doubtless present in hypoxic-ischaemic cases, although speech does appear to be a resistant function (Lu-Emerson $\&$ Khot, 2010). For example, incontinence has been reported in rare cases of posthypoxic demyelination (Thacker, Asthana \& Sarkari, 1995). 
Perhaps Table 2 might be most helpfully seen as a preliminary attempt at providing a general profile of strangulation outcomes. As such it spotlights the severity of the crime.

Strangulation results in the majority of expected clinical outcomes of cardiac arrest, hanging, carbon monoxide poisoning, or drowning, but with additional trauma-based psychological distress, significant neurological damage, behavioural change, and the risk of miscarriage. Despite this severity, the data suggest strangulation victims may receive less intervention, partly due to delayed symptom development, and the observation that as few as $5 \%$ of victims seek medical assistance (Strack et al., 2001).

\section{Discussion}

Our systematic literature search identified 27 empirical, peer-reviewed studies which documented the outcomes of non-fatal strangulation in IPV and sexual assault. Almost all victims were female. 18 studies reported on the pathology of the attack, which included severe, life-threatening injuries: delayed stroke, arterial bisection, basal ganglia, frontal lobe, and cerebellar lesions, hyoid fractures, tracheal perforations, and signs of venous congestion, including petechial haemorrhage. 23 described the neurological outcomes, with loss of consciousness reported in up to $38 \%$ of strangulation events (Shields et al., 2010), strongly suggestive of acquired brain injury, and other serious sequelae including changes to vision and voice, limb weakness and paralysis, sensory loss, and movement disorder. 17 studies described the psychological outcomes, indicative of a profound trauma reaction: PTSD, existential fear, depression, anxiety, suicidality, personality change, insomnia, dissociation, and shame. Markedly fewer studies reported on cognitive and behavioural changes. Amnesia for events was mentioned in six papers, and one was suggestive of executive difficulties. One study described subsequent compliant and submissive behaviour, and two reported 
survivalism and aggression towards the attacker. Five studies drew attention to the lack of help-seeking behaviour by the victims, even when there had been multiple strangulation attempts. There were no studies conducted from a neuropsychological perspective: the majority of papers found in our search were hospital-based case reports, or retrospective analyses of police and legal records. As such, they tended to focus on physical and visible (including via imaging) injury.

It was unfortunate that the one study identified in the course of the search which most precisely answered the research question had to be excluded: it was a doctoral thesis, and had not been peer-reviewed (Pierquet, 1997). In her study, Pierquet administered a comprehensive neuropsychological battery with 45 women who had endured IPV. She recorded data on the types of violence suffered, including whether they had been 'choked', if this had been to the point of unconsciousness $(13 / 45,29 \%$ of the sample), and frequency (up to 10). From this, Pierquet was able to demonstrate a significant association between strangulation and cerebral dysfunction, including memory, cognitive flexibility, and motor deficits $($ Eta squared $=0.549)$. She was also able to evidence a dose effect, in that the amount of times someone was strangled accounted for almost a third of the variance in cognitive and motor function.

Another, sadly unpublished, thesis with alarming results demonstrated a 10-15 fold increased risk of stroke amongst women under 40 who had suffered IPV $(n=237)$ versus a matched control (Smith, 2009). 73\% of the sample had been strangled; only 3\% of those had been admitted to hospital. Smith demonstrated significant associations between strangulation, loss of memory, paralysis, and stroke. Combining this with the incidence figures for strangulation gives substance to the warnings elsewhere in the literature that strangulation may be the second most common cause of stroke in women under 40, and should be included in the 
differential diagnosis of younger patients (Malek et al., 2000). Seven papers we found diagnosed haemorrhagic or ischaemic stroke, often significantly delayed.

This systematic review highlights four main points. Firstly, that strangulation within IPV and sexual assault is a highly gendered crime. Secondly, that strangulation shares all the consequences of hypoxic-ischaemic injury, such as cardiac arrest, but can have additional, severe neurological, psychological, and behavioural outcomes, many of which are due to the highly traumatising nature of this particularly intimate terrorism (Johnson, 2010). Thirdly, that these women are unlikely to present to healthcare services, despite the severity of their injuries. Finally, and related to our original questions, that, despite growing interest, we still do not know enough about the neuropsychological outcomes of strangulation in IPV and sexual assault, and that therefore potentially those women who do present to medical settings are being discharged with no real understanding of long-term consequences, nor receiving appropriate neuropsychological intervention.

This last point is important, because we need this evidence base in order to build public and institutional understanding of the gravity of strangulation. In a recent British legal case, a prostitute had been strangled by a client (Armstrong, 2012). With her losing consciousness, he had panicked, believed her to be dead, and was in the process of abandoning her body by the roadside when she regained consciousness. The victim then went to her attacker's house, where they drank wine together. Her behaviour after the event was used to undermine the severity of the attack, he was found guilty of the lesser charge of grievous bodily harm, and sentenced to two years. Based on the literature we have reviewed, her behaviour could have been due to existential fear, and therefore displaying compliance in order to survive. Having lost consciousness she would almost certainly be amnesic for that portion of the attack, but she could also have wider retrograde memory loss. It could be the result of damage to brain areas involved in executive function - she could not problem-solve or plan an escape - and 
general hypoxic confusion. But if none of this is systematically evidenced, then victims' behaviour will not be seen as the product of a strangled brain.

This lack of appreciation for the severity of strangulation has other far-reaching consequences. The campaign group We Can’t Consent To This (https://wecantconsenttothis.uk/) has collated 250 cases of women fatally strangled by men during sexual intercourse, in which the legal defence team argued that the victim consented, that therefore the death was accidental, and consequently the assailant not guilty of murder. But from a neuropsychological perspective, cognitively consent hinges on two factors: it must be informed, and there needs to be capacity to withdraw it at any point. If strangulation - its mechanics, its severity - is not understood, then the victim is not informed. The Red Wing studies (Kabat \& Anderson, 1943) undermine the second point. The prisoners and psychiatric inmates who were fitted with the mechanical strangulation cuffs were also given an emergency release button. The lead investigator, when testing the equipment on himself, described being close to losing consciousness and finding himself unable to press the button (Smith, Clayton \& Robertson, 2011). The potential onset of dyspraxia, amnesia, and unconsciousness itself (in as little as four seconds) are disabling: the very organ that is needed to withdraw consent is compromised by the activity to which that consent applies. The term 'consenting kink' is therefore a potentially fatal misnomer.

This is worrying, in the context of strangulation having become normalised (Edwards, 2016). In a recent UK survey $(\mathrm{N}=2,002) 38 \%$ of women under 40 had experienced strangulation during sex, with $42 \%$ of those saying it was unwanted, and that they had felt pressured, coerced, or forced (BBC, 2019). Strangulation is a pornographic trope, and features within popular mainstream literature, such as the Fifty Shades series. At the time of writing this paper the Google search algorithms would autocomplete 'choke' with 'someone safely' and '... ga girl meme', and online shops to buy accessories for 'breath play' are promoted. A recent 
systematic review calculated a median $7.4 \%$ prevalence amongst teenagers for the 'choking game', in which ligatures are used to strangle oneself, with this filmed and shared on social media, and 99 deaths reported in the literature (Busse, Harrop, Gunell, Kipping, 2015). Although many police forces have abandoned the use of carotid restraint following deaths in custody, it is still used in many countries, and by the military (Stellpflug, Menton, Corry, \& Schneir, 2020). 'Near chokes', 'chokes', and 'submission holds' are also legitimate and widely utilised tactics in mixed martial arts (MMA).

To put this into context, waterboarding has now been internationally outlawed as a form of torture, correctly considered inhumane and unacceptably dangerous, even when its stated objective is to prevent multiple deaths. In waterboarding, however, it is only the airway which is occluded. Strangulation is more lethal: not only is breathing interrupted, but also blood flow to and from the brain. We have shown how it can carry all the consequences of hypoxic-ischaemic injury such as cardiac arrest, and more besides. There is something societally flawed about banning the waterboarding of terrorists, whilst ignoring the intimate terrorism (Johnson, 2010) of those millions of women around the world who are regular victims of strangulation.

\section{Review limitations}

The neuropsychological sequelae of strangulation is an embryonic field, it straddles different disciplines, and multiple terms are used to describe strangulation. Despite our best efforts, it is therefore extremely likely that our search missed studies. In those papers we did find, few controlled for confounding factors. It was therefore sometimes difficult to separate out what outcomes were specific to strangulation as opposed to the general traumatising effects of IPV and sexual assault. This is particularly relevant for those instances where PTSD was reported, given associations have been demonstrated elsewhere between PTSD and hippocampal 
volume, speed of processing, and reasoning performance (Twamley, Allard, Thorp \& Norman, 2009). Finally, the lack of consistent, validated assessment tools rendered betweenstudy comparisons difficult, and meta-analysis impossible.

\section{Further research}

In our opinion, it is imperative that new, peer-reviewed studies add to our neuropsychological understanding of strangulation, by investigating the cognitive, psychological, and behavioural outcomes, measured with standardised assessment tools, set against control groups. This will help in terms of isolating the effects of strangulation. It will also be important to recruit from non-hospital or IPV settings (given the majority of victims do not seek assistance). This lack of help-seeking behaviour merits exploration in its own right. The majority of studies were from the USA: does this pattern still exist in countries where healthcare is free at the point of need? What barriers prevent women from presenting? For those who do receive intervention, although it was not the objective of this search, little was found in the literature which evidences best practice and treatment outcomes for holistic, long-term recovery, beyond acute medical care.

Again, although not the aim of this study, the societal normalisation of strangling we observed is worrying. There would be merit in trying to understand the attraction of, and possible pressure to partake in, 'breath play' and the 'choking game'. Similarly, the use of 'chokes' (loss of consciousness) and 'submission holds' (in which respiration is blocked) in MMA is extremely concerning. Other sports, based on the evidence, have banned repeated heading of balls by young people, given the cumulative effect of multiple concussions on cognitive function, and the future risk of developing chronic traumatic encephalopathy (Stein, Alvarez \& McKee, 2014). There are emerging case reports and studies on choking and carotid injury in MMA (Lim, Ho \& Ho, 2019; Powell et al., 2018). There was a recent 
systematic review on TBI in MMA (Lockwood, Frame, Lin \& Ackerley, 2018), but it focused on 'knockouts' rather than strangulation.

One of the strangulation mechanisms described in the Introduction is thyroid storm: thyrocytosis resulting from damage to the gland, which can cause multiple organ failure, including lethal arrhythmias. Malek et al. (2001) reported hyperthyroidism in their cases but stated this was pre-morbid. Given hyperthyroidism's non-fatal effects can include anxiety, insomnia, and several other strangulation outcomes attributed to the psychological impact, there may be merit in exploring further this interaction, as its incidence may be underreported.

Finally, the high incidence of miscarriage and delayed stroke were shocking. Further epidemiological investigation could be conducted to substantiate a call to healthcare providers to consider strangulation a differential diagnosis for both events. These could be rare and critical moments in which to identify IPV victims, who may have no other physical signs of strangulation, and provide life-saving intervention.

\section{Conclusion}

This systematic review found 27 empirical, peer-reviewed studies which together evidence the severe outcomes of strangulation within IPV and sexual assault. Given the mechanisms, involving potential occlusion of the airway, blood flow to and from the brain, and the triggering of the carotid sinus reflex, the neurological consequences can include all those associated with hypoxic-ischaemic injury, such as cardiac arrest. But there are other psychological outcomes linked to this uniquely intimate terrorism and its traumatising nature: the pain of watching "the man who so-called loves you try to kill you" (Thomas et al., 2014, p. 130). The majority of studies we found were based on hospital case reports, or existing police and legal records. At present there is less evidence for strangulation's cognitive and 
behavioural sequelae, and none based on objective, neuropsychological testing. There is therefore a need to build the evidence base, and this work should use control groups, and standardised assessment tools.

Perhaps more importantly, however, is the need to use the science to inform institutions and the public; to reposition strangulation from being a game, to serious criminal assault. Othello believed that Justice would be breaking her sword if he did not strangle Desdemona. In our view, Justice needs to keep her sword firmly raised until the millions of Othellos and Desdemonas around the world understand, as our review of the evidence shows, that strangulation - even for seconds - can cause lifechanging damage to the mind, and the brain.

Acknowledgement: With thanks to Yasmin Noorani at Bangor University Library for her advice on search strategy.

Disclosure of interests: The authors report no conflict of interest.

Word count: 11,967 with references, 9,760 without 


\section{References}

Adolfsson A. (2011). Meta-analysis to obtain a scale of psychological reaction after perinatal loss: focus on miscarriage. Psychology Research and Behavior Management, 4, 29-39.

Archer, J. (2002). Sex differences in physically aggressive acts between heterosexual partners: A meta-analytic review. Aggression and Violent Behavior, 7(4), 313-351.

Arciniegas, D. (2012). Hypoxic-Ischaemic Brain Injury. International Brain Injury Association. Retrieved from https://www.internationalbrain.org/articles/hypoxicischemic-brain-injury/.

Armstrong, (2012). Man choked prostitute after watching Billie Piper in Secret Diary of a Call Girl. The Mirror. Retrieved from https://www.mirror.co.uk/news/uk-news/man-choked-prostitute-after-watching-145613

Bagwell-Gray, M. E., Messing, J. T., \& Baldwin-White, A. (2015). Intimate partner sexual violence: A review of terms, definitions, and prevalence. Trauma, Violence, \& Abuse, 16(3), 316-335.

Banks, M. E. (2007). Overlooked but critical: Traumatic brain injury as a consequence of interpersonal violence. Trauma, Violence, \& Abuse, 8(3), 290-298.

Banzett, R. B., Lansing, R. W., Evans, K. C., \& Shea, S. A. (1996). Stimulus-response characteristics of CO2-induced air hunger in normal subjects. Respiration Physiology, 103(1), 19-31.

British Broadcasting Corporation (2019). Savanta ComRes survey. Table with full results retrieved from https://www.comresglobal.com/wp-content/uploads/2019/11/Final-BBC-5-Live-Tables_211119cdh.pdf

Busse H, Harrop T, Gunnell D, et al. Prevalence and associated harm of engagement in self-asphyxial behaviours ('choking game') in young people: A systematic review. Archives of Disease in Childhood, 100, 1106-1114

Cameron, R. P., \& Gusman, D. (2003). The primary care PTSD screen (PC-PTSD): development and operating characteristics. Primary Care Psychiatry, 9(1), 9-14.

Campbell, J. C., Webster, D. W., \& Glass, N. (2009). The danger assessment: Validation of a lethality risk assessment instrument for intimate partner femicide. Journal of Interpersonal Violence, 24(4), 653-674.

Chapman, J. C., \& Diaz-Arrastia, R. (2014). Military traumatic brain injury: a review. Alzheimer's \& Dementia, 10, S97S104.

Clarot, F., Vaz, E., Papin, F., \& Proust, B. (2005). Fatal and non-fatal bilateral delayed carotid artery dissection after manual strangulation. Forensic Science International, 149(2-3), 143-150.

Cooper, I. S. (1949). Medical Aspects of the Death of Desdemona. Postgraduate Medicine, 5(6), 520-525.

Corrigan, J. D., Wolfe, M., Mysiw, W. J., Jackson, R. D., \& Bogner, J. A. (2003). Early identification of mild traumatic brain injury in female victims of domestic violence. American Journal of Obstetrics and Gynaecology, 188(5), S71-S76. 
Davins-Pujols, M., Salamero, M., Aznar-Martínez, B., Aramburu-Alegret, I., \& Pérez-Testor, C. (2014). Acts of intimate partner violence and feelings of danger in battered women seeking help in a Spanish specialized care unit. Journal of Family Violence, 29(7), 703-712.

De Boos, J. (2019). Tracheal perforation from non-fatal manual strangulation. Journal of Forensic and Legal Medicine, $66,1-3$.

Edwards, S. (2016). Assault, strangulation and murder - challenging the sexual libido consent defence narrative, in A. Reed \& M. Bohlander, with N. Wake \& E. Smith (eds), Consent: Domestic and Comparative Perspectives. Abingdon, UK: Routledge.

Eisikovits, Z., \& Winstok, Z. (2002). Reconstructing intimate violence: The structure and content of recollections of violent events. Qualitative Health Research, 12(5), 685-699.

Farr, K. A. (2002). Battered women who were "being killed and survived it": Straight talk from survivors. Violence and Victims, 17(3), 267-281.

Funk, M., \& Schuppel, J. (2003). Strangulation injuries. WMJ-MADISON-, 102(3), 41-45.

Glass, N., Laughon, K., Campbell, J., Block, C. R., Hanson, G., Sharps, P.W., \& Taliaferro, T. (2008). Non-fatal strangulation is an important risk factor for homicide of women. The Journal of Emergency Medicine, 35(3), $329-335$

Green, S. (2017). Strangulation is a powerful tool of violence that often leaves no physical mark. Training Institute on Strangulation Prevention. Retrieved at: https://www.strangulationtraininginstitute.com/strangulation-is-apowerful-tool-of-violence-that-often-leaves-no-physical-mark-officials-in-king-county-want-it-noticedprosecuted/

Hawley, D. A., McClane, G. E., \& Strack, G. B. (2001). A review of 300 attempted strangulation cases part III: injuries in fatal cases. The Journal of Emergency Medicine, 21(3), 317-322.

Joanna Briggs Institute (2017). Critical Appraisal Tools. Retrieved from https://joannabriggs.org/ebp/critical_appraisal_tools

Johnson, M. P. (2010). A typology of domestic violence: Intimate terrorism, violent resistance, and situational couple violence. Northeastern University Press: Upne.

Joshi, M., Rahill, G. J., Lescano, C., \& Jean, F. (2014). Language of sexual violence in Haiti: perceptions of victims, community-level workers, and health care providers. Journal of Health Care for the Poor and Underserved, 25(4), 1623-1640.

Joshi, M., Thomas, K. A., \& Sorenson, S. B. (2012). “I didn't know I could turn colors”: Health problems and health care experiences of women strangled by an intimate partner. Social Work in Health Care, 51(9), 798-814.

Kabat, H., \& Anderson, J. P. (1943). Acute arrest of cerebral circulation in man: Lieutenant Ralph Rossen. Archives of Neurology \& Psychiatry, 50(5), 510-528. 
Koh, J. O., Cassidy, J. D., \& Watkinson, E. J. (2003). Incidence of concussion in contact sports: a systematic review of the evidence. Brain Injury, 17(10), 901-917.

Kwako, L. E., Glass, N., Campbell, J., Melvin, K. C., Barr, T., \& Gill, J. M. (2011). Traumatic brain injury in intimate partner violence: A critical review of outcomes and mechanisms. Trauma, Violence, \& Abuse, 12(3), 115-126.

Le Blanc-Louvry, I., Papin, F., Vaz, E., \& Proust, B. (2013). Cervical arterial injury after strangulation-different types of arterial lesions. Journal of Forensic Sciences, 58(6), 1640-1643.

Levack, M. M., Pettitt, B. J., \& Winston, A. D. (2009). Carotid artery thrombosis and delayed stroke associated with the use of a shoulder belt in a teenager. Journal of Pediatric Surgery, 44(8), e29-e33.

Lim, L. J., Ho, R., \& Ho, C. S. (2019). Dangers of mixed martial arts in the development of chronic traumatic encephalopathy. International journal of environmental research and public health, 16(2), 254.

Lockwood, J., Frape, L., Lin, S., \& Ackery, A. (2018). Traumatic brain injuries in mixed martial arts: A systematic review. Trauma, 20(4), 245-254.

Lu-Emerson, C., \& Khot, S. (2010). Neurological sequelae of hypoxic-ischemic brain injury. NeuroRehabilitation, 26(1), $35-45$.

Malec, J. F., Brown, A. W., Leibson, C. L., Flaada, J. T., Mandrekar, J. N., Diehl, N. N., \& Perkins, P. K. (2007). The Mayo classification system for traumatic brain injury severity. Journal of Neurotrauma, 24(9), 1417-1424.

Malek, A. M., Higashida, R. T., Halbach, V. V., Dowd, C. F., Phatouros, C. C., Lempert, T. E., ... \& Stoney, R. (2000). Patient presentation, angiographic features, and treatment of strangulation-induced dissection of the internal carotid artery: Report of three cases. Journal of Neurosurgery, 92(3), 481-487.

McClane, G. E., Strack, G. B., \& Hawley, D. (2001). A review of 300 attempted strangulation cases Part II: clinical evaluation of the surviving victim. The Journal of emergency medicine, 21(3), 311-315.

Mcquown, C., Frey, J., Steer, S., Fletcher, G. E., Kinkopf, B., Fakler, M., \& Prulhiere, V. (2016). Prevalence of strangulation in survivors of sexual assault and domestic violence. The American journal of emergency medicine, 34(7), 1281-1285.

Meel, B. L. (2015). A case report on near manual strangulation and Glasgow coma scale. African Health Sciences, 15(3), 1038-1040.

Messing, J. T., Patch, M., Wilson, J. S., Kelen, G. D., \& Campbell, J. (2018). Differentiating among attempted, completed, and multiple nonfatal strangulation in women experiencing intimate partner violence. Women's Health Issues, 28(1), 104-111.

Messing, J. T., Thaller, J., \& Bagwell, M. (2014). Factors related to sexual abuse and forced sex in a sample of women experiencing police-involved intimate partner violence. Health \& Social Work, 39(3), 181-191.

Miao, J., Su, C., Wang, W., Lin, H., Li, H., Lei, G., ... \& Li, Z. (2009). Delayed parkinsonism with a selective symmetric basal ganglia lesion after manual strangulation. Journal of Clinical Neuroscience, 16(4), 573-575. 
Milligan, N., \& Anderson, M. (1980). Conjugal disharmony: a hitherto unrecognised cause of strokes. $\mathrm{Br} M e d \mathrm{~J}$, 281(6237), 421-422.

Mittal, M., Resch, K., Nichols-Hadeed, C., Stone, J. T., Thevenet-Morrison, K., Faurot, C., \& Cerulli, C. (2018). Examining associations between strangulation and depressive symptoms in women with intimate partner violence histories. Violence and Victims, 33(6), 1072-1087.

Moher, D., Liberati, A., Tetzlaff, J., \& Altman, D. G. (2009). Preferred reporting items for systematic reviews and metaanalyses: the PRISMA statement. Annals of Internal Medicine, 151(4), 264-269.

Monahan, K., Purushotham, A., \& Biegon, A. (2019). Neurological implications of nonfatal strangulation and intimate partner violence. Future Neurology, 14(3), FNL21.

Patch, M., Anderson, J. C., \& Campbell, J. C. (2018). Injuries of women surviving intimate partner strangulation and subsequent emergency health care seeking: an integrative evidence review. Journal of Emergency Nursing, 44(4), 384-393.

Pierquet, L. (1997). Neuropsychological consequences of the domestic battering of women. Doctoral thesis submitted to the Wisconsin School of Professional Psychology. Retrieved via Inter-Library Loan from UMI, Ann Arbor, Michigan.

Plattner, T., Bolliger, S., \& Zollinger, U. (2005). Forensic assessment of survived strangulation. Forensic Science International, 153(2-3), 202-207.

Powell, T., Fullam, T., Hammett, J., Nettlow, D., \& Harris, J. (2018). Vertebral Artery Dissection in Active-Duty Soldier Due to Mixed Martial Arts Choke Hold. Federal Practitioner, 35(7), 12.

Pritchard, A. J., Reckdenwald, A., \& Nordham, C. (2017). Nonfatal strangulation as part of domestic violence: A review of research. Trauma, Violence, \& Abuse, 18(4), 407-424.

Pritchard, A. J., Reckdenwald, A., Nordham, C., \& Holton, J. (2018). Improving identification of strangulation injuries in domestic violence: Pilot data from a researcher-practitioner collaboration. Feminist Criminology, 13(2), 160181.

Purvin, V. (1997). Unilateral headache and ptosis in a 30-year-old woman. Survey of Ophthalmology, 42(2), $163-168$.

Radloff, L. S. (1977). The CES-D scale: A self-report depression scale for research in the general population. Applied Psychological Measurement, 1(3), 385-401.

Ralston, B., Rable, J., Larson, T., Handmaker, H., \& Lifshitz, J. (2019). Forensic nursing examination to screen for traumatic brain injury following intimate partner violence. Journal of Aggression, Maltreatment \& Trauma, $28(6), 732-743$.

Rosenberg, M. (1965). Rosenberg self-esteem scale (RSE). Acceptance and commitment therapy: Measures package, $61(52), 18$. 
Shields, L. B., Corey, T. S., Weakley-Jones, B., \& Stewart, D. (2010). Living victims of strangulation: a 10-year review of cases in a metropolitan community. The American Journal of Forensic Medicine and Pathology, 31(4), 320325.

Smith, Y. (2009). Exploring psychosocial risk factors for stroke in young women exposed to domestic violence.

Dissertation submitted to Queen Margaret University, UK. Retrieved from http://europepmc.org/article/ETH/500505.

Smith, B. A., Clayton, E. W., \& Robertson, D. (2011). Experimental arrest of cerebral blood flow in human subjects: the red wing studies revisited. Perspectives in Biology and Medicine, 54(2).

Smith, P. H., Earp, J. A., \& DeVellis, R. (1995). Measuring battering: development of the Women's Experience with Battering (WEB) Scale. Women's Health: Research on Gender, Behavior, \& Policy.

Smith Jr, D. J., Mills, T., \& Taliaferro, E. H. (2001). Frequency and relationship of reported symptomology in victims of intimate partner violence: The effect of multiple strangulation attacks. The Journal of Emergency Medicine, 21(3), 323-329.

Sorenson, S. B., Joshi, M., \& Sivitz, E. (2014). A systematic review of the epidemiology of nonfatal strangulation, a human rights and health concern. American Journal of Public Health, 104(11), e54-e61.

Stein, T. D., Alvarez, V. E., \& McKee, A. C. (2014). Chronic traumatic encephalopathy: a spectrum of neuropathological changes following repetitive brain trauma in athletes and military personnel. Alzheimer's Research \& Therapy, 6(1), 4.

Stellpflug, S. J., Menton, T. R., Corry, J. J., \& Schneir, A. B. (2020). There is more to the mechanism of unconsciousness from vascular neck restraint than simply carotid compression. International Journal of Neuroscience, 130(1), 103-106.

Strack, G. B., \& Gwinn, C. (2011). On the edge of homicide: Strangulation as a prelude. Crim. Just., 26(32).

Strack, G. B., McClane, G. E., \& Hawley, D. (2001). A review of 300 attempted strangulation cases Part I: Criminal legal issues. The Journal of Emergency Medicine, 21(3), 303-309.

Straus, M. A., Hamby, S. L., Boney-McCoy, S., \& Sugarman, D. B. (1996). The revised conflict tactics scales (CTS2) development and preliminary psychometric data. Journal of Family Issues, 17(3), 283-316.

Taliaferro, E., Mills, T., \& Walker, S. (2001). Walking and talking victims of strangulation. Is there a new epidemic? A commentary. The Journal of Emergency Medicine, 21(3), 293-295.

Thacker, A. K., Asthana, A. B., \& Sarkari, N. B. S. (1995). Delayed post-anoxic encephalopathy. Postgraduate Medical Journal, 71(836), 373.

Thomas, K. A., Joshi, M., \& Sorenson, S. B. (2014). “Do you know what it feels like to drown?” Strangulation as coercive control in intimate relationships. Psychology of Women Quarterly, 38(1), 124-137. 
Twamley, E. W., Allard, C. B., Thorp, S. R., Norman, S. B., Cissell, S. H., Berardi, K. H., ... \& Stein, M. B. (2009). Cognitive impairment and functioning in PTSD related to intimate partner violence. Journal of the International Neuropsychological Society, 15(6), 879-887.

Vella, S. A. (2013). Cognitions and behaviors of strangulation survivors of intimate terrorism. Alliant International University.

White, C., \& Majeed-Ariss, R. (2018). Non-fatal strangulation amongst clients attending Saint Mary’s SARC. Manchester University NHS Foundation Trust. Retrieved from https://www.stmaryscentre.org/application/files/5815/6439/0323/White2018.pdf.

Wilbur, L., Higley, M., Hatfield, J., Surprenant, Z., Taliaferro, E., Smith Jr, D. J., \& Paolo, A. (2001). Survey results of women who have been strangled while in an abusive relationship. The Journal of Emergency Medicine, 21(3), 297-302.

World Health Organisation (2019). Violence against women - Evidence brief. Retrieved from https://www.who.int/reproductivehealth/publications/vaw-evidence-brief/en/.

Yen, K., Vock, P., Christe, A., Scheurer, E., Plattner, T., Schön, C., ... \& Dirnhofer, R. (2007). Clinical forensic radiology in strangulation victims: forensic expertise based on magnetic resonance imaging (MRI) findings. International Journal of Legal Medicine, 121(2), 115-123.

Zilkens, R. R., Phillips, M. A., Kelly, M. C., Mukhtar, S. A., Semmens, J. B., \& Smith, D. A. (2016). Non-fatal strangulation in sexual assault: A study of clinical and assault characteristics highlighting the role of intimate partner violence. Journal of Forensic and Legal Medicine, 43, 1-7.

Zink, T., Klesges, L. M., Levin, L., \& Putnam, F. (2007). Abuse behavior inventory: Cutpoint, validity, and characterization of discrepancies. Journal of Interpersonal Violence, 22(7), 921-931. 